\title{
Spherical $f$-Tilings by Two Noncongruent Classes of Isosceles Triangles
}

\author{
${ }^{123}$ A. M. d'Azevedo Breda, Robert Dawson and Patrícia S. Ribeiro \\ ${ }^{1}$ Department of Mathematics \\ University of Aveiro3810-193 Aveiro, Portugal \\ ambreda@ua.pt \\ ${ }^{2}$ Department of Mathematics and Computing Science \\ Saint Mary's University, \\ Halifax, Nova Scotia, B3H 3C3 Canada \\ rdawson@cs.stmarys.ca \\ ${ }^{3}$ Department of Mathematics \\ E.S.T. Setúbal 2910-761 Setúbal, Portugal \\ patricia.ribeiro@estsetubal.ips.pt
}

\section{Abstract}

The study of spherical dihedral $f$-tilings when the prototiles are two noncongruent isosceles triangles was started in two previous papers. Here, we complete the classification, characterizing the $f$-tilings that satisfy the remaining case of adjacency. As it will be shown, this class is composed by two three-parameter families denoted by $\mathrm{A}^{n, m, \delta}$ and $\mathrm{B}^{n, m, \delta}$ and a related four-parameter family denoted by $\mathrm{B}^{p, q, p^{\prime}, q^{\prime}}$, where $p>p^{\prime} \geq 0, q^{\prime}>q \geq 0$; two isolated tilings denoted by $\mathrm{P}$ and $\mathrm{Q}$; and six distinct discrete families denoted by $\mathrm{J}^{k}, \mathrm{~L}^{k}, \mathrm{M}^{k}, \mathrm{~N}^{k}(k \geq 3)$; and $\mathrm{F}^{k}, \mathrm{~K}^{k}$ with $k \geq 4$.

MSC 2010: 52C20, 05B45, 52C30

\section{Keywords: dihedral $f$-tilings; spherical triangulations; spherical trigonometry}

\section{Introduction}

Spherical folding tilings or $f$-tilings for short, are edge-to-edge tilings of the Euclidian sphere $S^{2}$, by geodesic polygons, such that all vertices are of even valency and both sums of alternate angles around each vertex is always $\pi$. Let us denote by $\Omega(X, Y)$ the set, up to isomorphism, of all dihedral f-tilings of $S^{2}$ whose cells are congruent to $X$ or $Y$. In [8], it was shown that any $\tau \in \Omega(X, Y)$ necessarily has vertices of valency four.

Some spherical $f$-tilings by congruent triangles, such as the spherical octahedron, have been known since antiquity; they were first specifically studied as a class in 1992 [2]. The researchers Yukako Ueno and Yoshio Agaoka have studied spherical tilings in depth: [11], [12]. In [1], the study of all spherical $f$-tilings by triangles and $r$-sided regular polygons, for any $r \geq 5$ was given. Dawson and Doyle also worked on spherical tilings with triangles, relaxing the edge to edge condition, [9], [10].

The classification of all dihedral spherical folding tilings by triangles is not yet completed. So far, the studied cases correspond to the following prototiles:

- an equilateral triangle and an isosceles triangle, [5];

- an equilateral triangle and a scalene triangle, [6].

- two noncongruent isosceles triangles in two types of adjacency (Figure 1-I, II), [3] and [4].

In this paper, we complete the classification of spherical $f$-tilings by two noncongruent isosceles triangles studying the last case of adjacency illustrated in Figure 1-III.

Let $T_{1}$ be an isosceles spherical triangle of angles $\alpha, \alpha, \beta$ with sides $a$ (oposite to $\alpha$ ) and $b$ (opposite to $\beta$ ), and $T_{2}$ another isosceles spherical triangle of angles $\gamma, \gamma, \delta$ with sides $c$ (opposite to $\gamma$ ) and $d$ (opposite to $\delta$ ).

There are three types of adjacency, based on which edges meet, as shown in the next figure; the third has two subcases depending on orientation. 


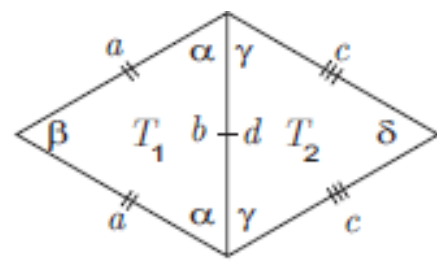

I

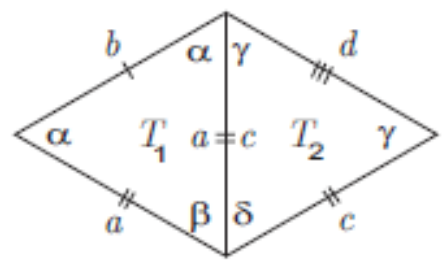

a)

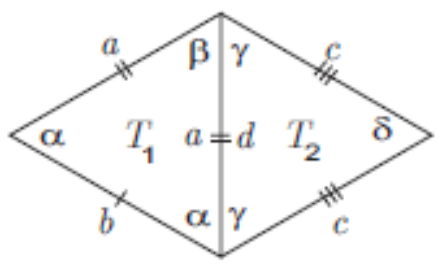

II

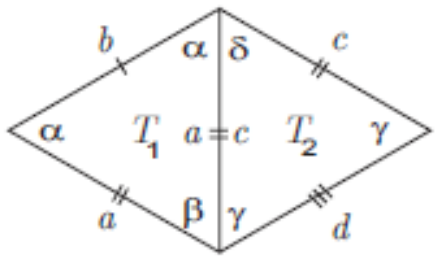

III

b)

Figure 1:Different types of adjacency.

Type III adjacency requires, by the spherical cosine law, that

which simplifies to

$$
\frac{\cos \alpha(1+\cos \beta)}{\sin \alpha \sin \beta}=\frac{\cos \gamma(1+\cos \delta)}{\sin \gamma \sin \delta},
$$

$$
\cot \alpha \cot \frac{\beta}{2}=\cot \gamma \cot \frac{\delta}{2} .
$$

In order to get any dihedral $f$-tiling $\tau \in \Omega\left(T_{1}, T_{2}\right)$, we find it useful to start by considering one of its local configurations, beginning with a vertex common to each one of the isosceles triangles in adjacent positions. In the diagrams that follows it is convenient to label the tiles according to the following procedures:

1. Tiles 1 and 2 are of type $T_{1}$ and $T_{2}$ respectively, and exhibit the specified adjacency;

2. For $j \geq 2$, unless it is specifically indicated that the tile location is hypothetical, the location of tile $j$ can be deduced from the positions of the tiles $(1,2, \ldots, j-1)$ and from the hypothesis that the configuration is part of a complete $f$-tiling.

\section{Triangular Dihedral $f$-Tilings by Isosceles Triangles}

In adjacency of type III, equation (1.2) shows that if $\beta=\delta$, then $\alpha=\gamma$ and the triangles are congruent, contrary to our assumption of dihedrality. As $\cot \left(\frac{\beta}{2}\right), \cot \left(\frac{\delta}{2}\right)>0$, the same equation shows that $\alpha$ and $\gamma$ are both right, both acute, or both obtuse. If $\alpha=\gamma \neq \frac{\pi}{2}$, this again implies tile congruence. Except in the right-angled case, we will consider, without loss of generality, $\alpha>\gamma$, whence, by Eq. (1.2), $\delta>\beta$. We shall make much use of the following result, which is a special case of Proposition 2.1 of [5].

Proposition 2.1 Every $f$-tiling of the sphere with triangles has at least six vertices of valency 4 .

In an $f$-tiling, the angles at a tetravalent vertex are each repeated twice and are supplementary. Moreover, in the cases we are now considering, a little more can be said.

Proposition 2.2 If a dihedral $f$-tiling has a pair of isosceles (nonequilateral) triangular tiles exhibiting adjacency of type III, two triangles of each type meet at each tetravalent vertex. If the tiling has more than 8 tiles, all tetravalent vertices have the same angles. 
Proof.Suppose (for instance) that $\alpha+\beta=\pi$, and that a tetravalent vertex $v_{1}$ using these angles exists (Figure 2-I). Then it is completely surrounded by tiles congruent to $T_{1}$. But $b$ is not equal to $a(=c)$; nor (as $a=c$ and $T_{1} \varsubsetneqq T_{2}$ ) to $d$; thus tile $\mathrm{A}$ is forced. But then the folding condition forces tile $\mathrm{B}$; and $v_{2}$ is also surrounded by copies of $T_{1}$. By induction, if the tiling can be completed it consists entirely of one type of tile, contradicting our assumption that it was dihedral. We conclude that one of the supplementary angles comes from each triangle.
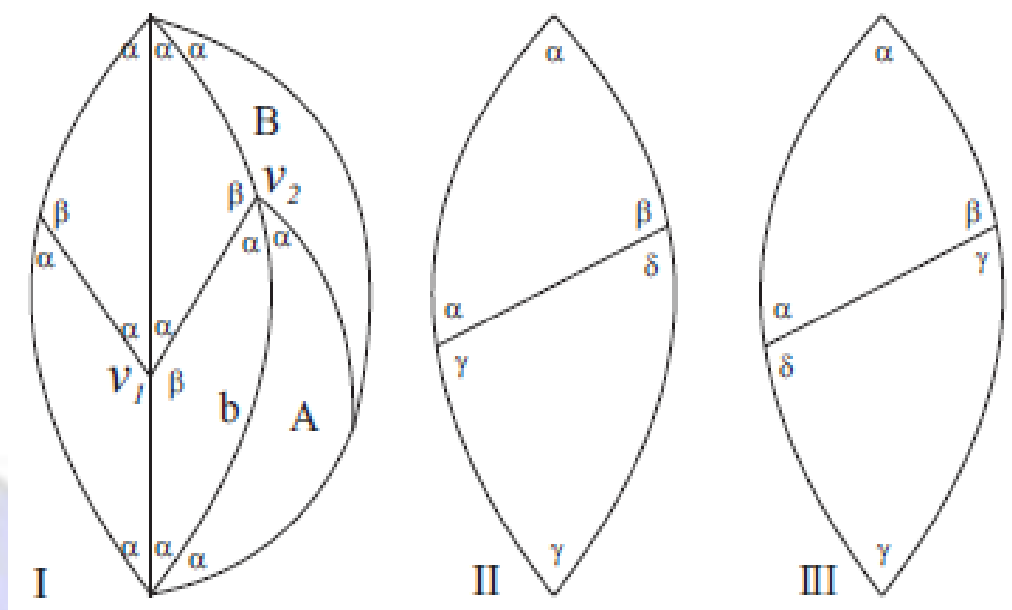

Figure 2:Configurations with tetravalent vertices

Clearly $\alpha$ (or $\beta$ ) is supplementary to $\gamma$, it cannot also be supplementary to $\delta$ (or $T_{2}$ would be equilateral.) This leaves the possibility of two disjoint pairs of supplementary angles: $\alpha+\delta=\gamma+\beta=\pi$ (Figure 2-II) or $\alpha+\gamma=\beta+\delta=\pi$ (Figure 2-III.)

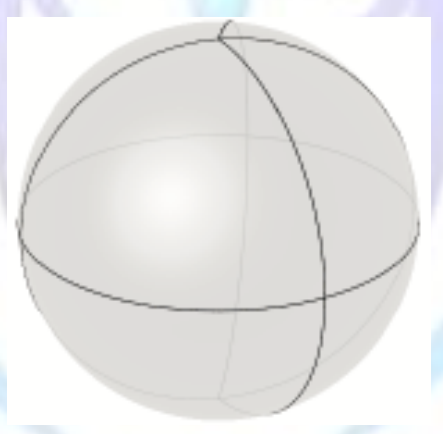

Figure 3:The tiling $A^{1,1 \delta}$.

In either case, $\alpha=\gamma$. In the first case, this makes $\beta=\delta$ and $T_{1} \cong T_{2}$, contrary to our assumptions. In the second case, $\alpha=\gamma=\pi / 2$ and the total area of the two triangles is $\pi$; it is easy to see that the tiling must be a member of the continuous family shown in Figure 3 , all of whose members have eight tiles. These tilings are part of the larger $A^{m, n, \delta}$ family that we will consider below; the other members, however, have only one species of tetravalent vertex.

\subsection{Tilings in which adjacency of type III a) is present}

We first consider the case in which triangles $T_{1}$ and $T_{2}$ appear somewhere in the tiling with an adjacency of type III a), as illustrated in Figure 4. In this adjacency, the triangles "point the same way" on the common edge 


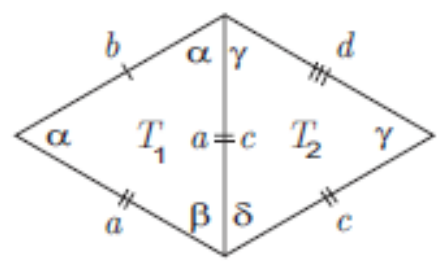

a)

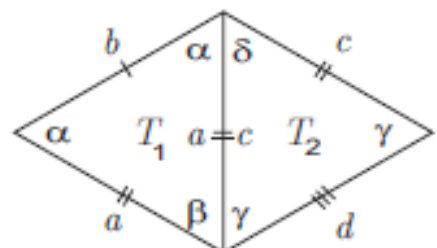

b)

Figure 4:Adjacency of type III.

Proposition 2.3 If $\gamma=\alpha=\frac{\pi}{2}$, we have precisely the following tilings:

1. For $m, n>0$, and arbitrary $\delta<\frac{\pi}{n}$, a finite set of tilings $\mathrm{A}^{n, m, \delta}$ with $\beta=\frac{\pi-n \delta}{m}$, each tiling containing $4(m+n)$ tiles arranged in two hemispherical rosettes (see Figure 5);

2. For $m, n>0$, and arbitrary $\delta<\frac{\pi}{n}$, a finite set of tilings $\mathrm{B}^{n, m, \delta}$ with $\beta=\frac{\pi-n \delta}{m}$, each tiling containing $4(m+n)$ tiles arranged in four half-rosettes (see Figure 6);

3. For $p>p^{\prime} \geq 0, q^{\prime}>q>0$, with $p+q<p^{\prime}+q^{\prime}$, a finite set of tilings $\mathrm{B}^{p, q, p^{\prime}, q^{\prime}}$ with $\beta=\frac{\left(q^{\prime}-q\right)}{2\left(q^{\prime} p-q p^{\prime}\right)} \pi, \gamma=\frac{\left(p-p^{\prime}\right)}{2\left(q^{\prime} p-q p^{\prime}\right)} \pi$, each tiling containing $4\left(p+q+p^{\prime}+q^{\prime}\right)$ tiles arranged in four halfrosettes (see Figure 7).
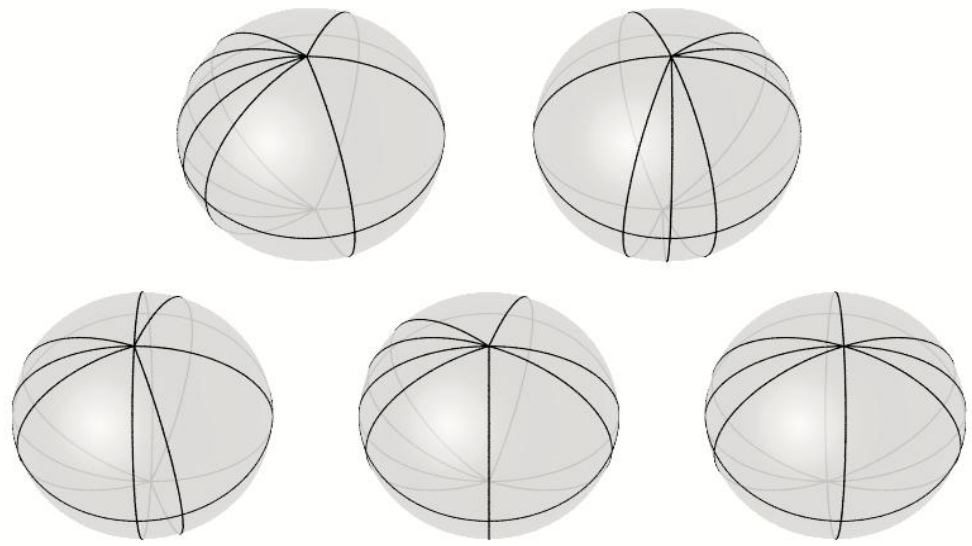

Figure 5:3D representations of the five $A^{2,2 \delta}$ tilings. 

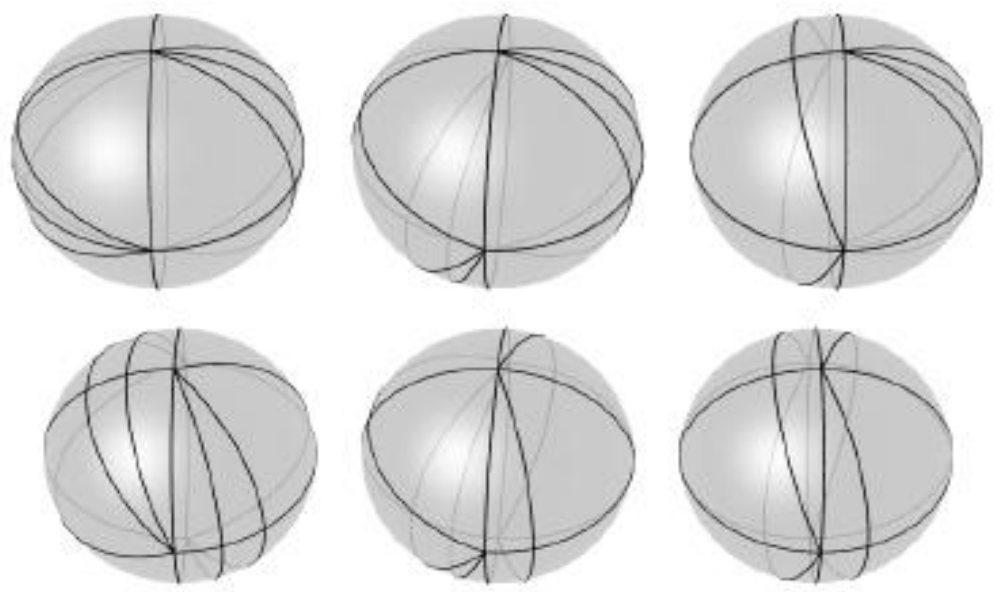

Figure 6:3D representations of the six $\mathrm{B}^{1,1 \delta}$ tilings.
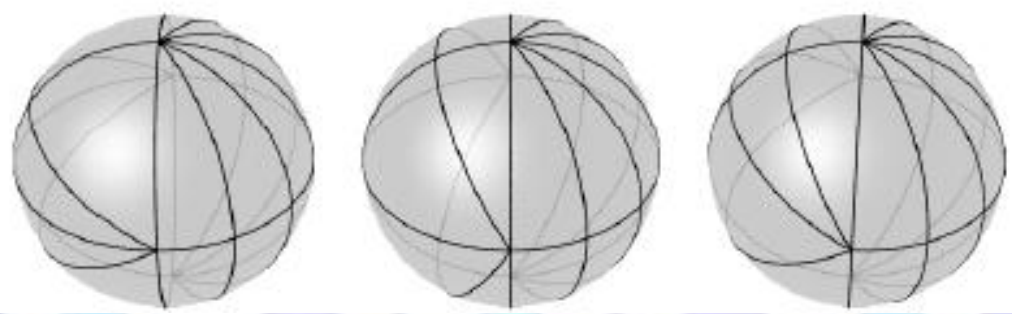

Figure 7:3D representations of the three $B^{1,1,0,3}$ tilings.

Remark 1 Within each class, the tilings differ only by the way that the triangles are arranged within the rosettes or halfrosettes. While enumerating these arrangements is a valid problem (related to the well-known "necklace" and "bracelet" enumeration problems), it is an essentially combinatorial problem rather than a geometric one, and we shall not give a solution here.

Remark 2 The tilings $\mathrm{B}^{n, m, \delta}$ and $\mathrm{B}^{p, q, p^{\prime}, q^{\prime}}$ are very similar; the first may be thought of as an underdetermined special case of the second.
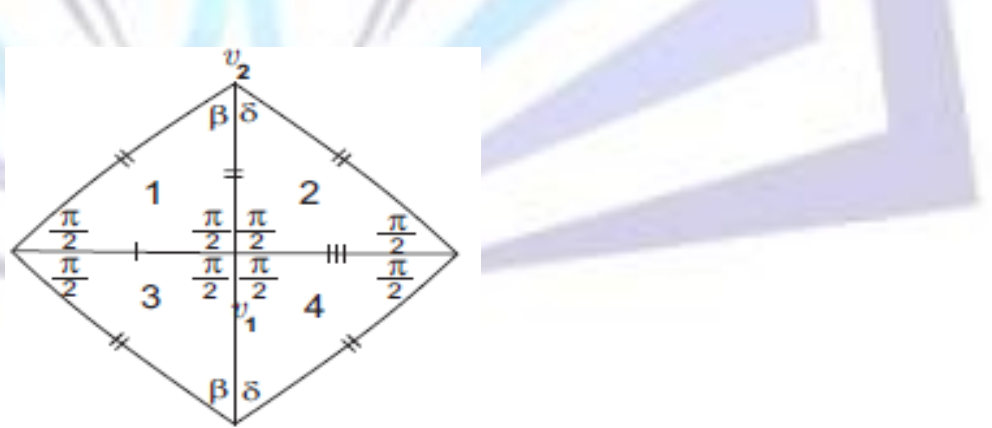

Figure 8:Local configuration at vertex $v_{1}$.

Proof.We have supposed that $\alpha=\gamma=\frac{\pi}{2}$. Without loss of generality, we will take $\beta>\delta$. Starting with two cells exhibiting type III a) adjacency, vertex $v_{1}$ (see Figure 8 ) is of valency four surrounded exclusively by angles $\frac{\pi}{2}$. Observe that if $\delta>\frac{\pi}{2}$, then necessarily $\beta>\frac{\pi}{2}$, and sums of alternate angles containing $\beta$ and $\delta$ do not satisfy the angle folding relation. 
The sum of alternate angles containing $\beta$, at vertex $v_{2}$ (see Figure 8 ), must be either of the form

$$
\begin{aligned}
& \text { - } m \beta+n \delta=\pi \text { or } \\
& \text { - } \frac{\pi}{2}+p \beta+q \delta=\pi,(p, q>0)
\end{aligned}
$$

Next, we shall study all cases separately.

Case A. The two sets of alternate angles have the same cardinality and the same sum; thus they have the same constituents. As there is, by hypothesis, more than one type of triangle, we have $m, n>0$. Every edge incident to a vertex at $v_{2}$ is of length $\pi / 2$; so the union of the triangles with vertices at $v_{2}$ covers one hemisphere, and edge matching forces the tiling in the other hemisphere to be a mirror image. The tiling therefore has no other vertex type with $\beta$ and $\delta$ angles, and no angle sum equation other than $m \beta+n \delta=\pi$ arises. The tiling is thus part of a continuous family with the same combinatorial structure but different angles. For each $(m, n, \delta)$, moreover, there are finitely many ways to arrange the tiles at the poles. We refer to any such tiling as being of type $A^{m, n, \delta}$. Each such tiling in the family has $4 m$ triangles congruent to $T_{1}$ and $4 n$ triangles congruent to $T_{2}$; they all have reflectional symmetry in the "equatorial plane" and differ only in their arrangements at the "poles".

Case B. Suppose that the sum of alternate angles containing $\beta$, at vertex $v_{2}$ is of the form $\frac{\pi}{2}+p \beta+q \delta=\pi,(p, q>0)$. Then there are exactly two edges of length $b$ or two of length $d$ at $v_{2}$, which must be paired; so the two right angles are adjacent. The other $2 p \beta$ angles and $2 q \delta$ angles form a "half-rosette". Matching the $b$ and $d$ edges of this forces a mirror-image half-rosette, completing one hemisphere. Now consider the vertex $v_{3}$ in figure 9; it has two right angles and a non-right angle. The sum of alternate angles there is thus either again of the form $\frac{\pi}{2}+p \beta+q \delta=\pi$; or of the form $\frac{\pi}{2}+p^{\prime} \beta+q^{\prime} \delta=\pi$. (As the adjacency condition has been satisfied at $v_{2}, p^{\prime}$ or $q^{\prime}$ may be 0.$)$
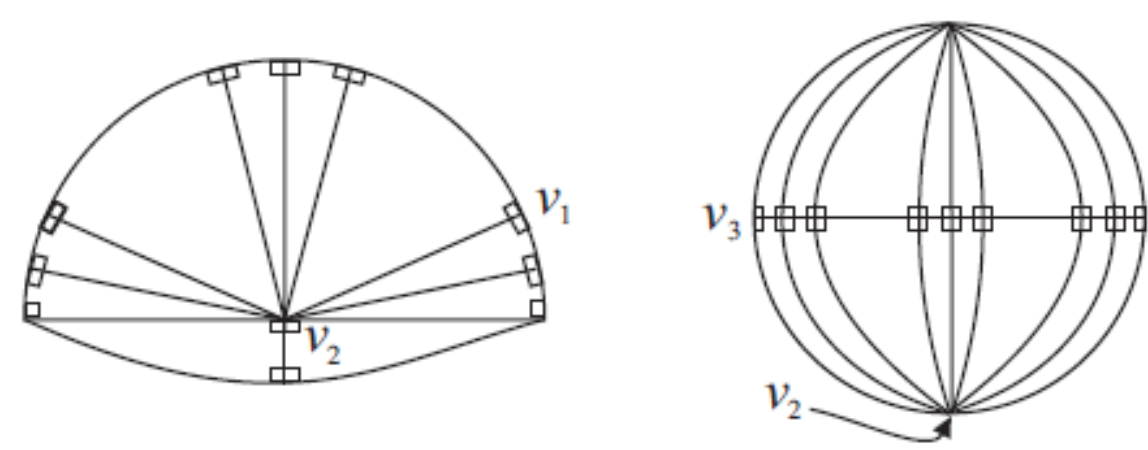

Figure 9:Local configurations when $\alpha=\gamma=\pi / 2$.

In the first case, we do not have enough independent constraints to determine $\beta$ and $\delta$, and the tiling is of the form $\mathrm{B}^{p, q, \delta}$. In the other case the system of equations $p \beta+q \delta=\pi / 2, p^{\prime} \beta+q^{\prime} \delta=\pi / 2$ has a unique solution, and the tiling is of the form $\mathrm{B}^{p, q, p^{\prime}, q^{\prime}}$. Not every set of parameters is possible; as $\beta>\delta>0$, we necessarily have $p>p^{\prime} \geq 0$, $q^{\prime}>q \geq 0$, and $p^{\prime}+q^{\prime}>p+q$. If these inequalities are satisfied, however, $q^{\prime} p-p^{\prime} q>0$ and $\beta=\frac{\left(q^{\prime}-q\right)}{2\left(q^{\prime} p-q p^{\prime}\right)} \pi$ and $\gamma=\frac{\left(p-p^{\prime}\right)}{2\left(q^{\prime} p-q p^{\prime}\right)} \pi$ are positive angles that tile as described.

Remark 3 Only the choice of adjacency type III a) forces the requirement that one hemisphere must contain both types of triangle, so that $q>0$. If we relax this requirement we still obtain tilings of type I and type III b) (vide infra.)

Assuming that $\alpha \neq \gamma$ and since $\alpha$ and $\gamma$ belong to the same quadrant, then in adjacency of type III a), we have 
$\alpha+\gamma<\pi$ (see Figure 10-I). Therefore, $\gamma<\alpha<\frac{\pi}{2}$ and consequently $\beta<\frac{\pi}{2} \leq \delta$ or $\frac{\pi}{2} \leq \beta<\delta$, since vertices of valency four must exist.
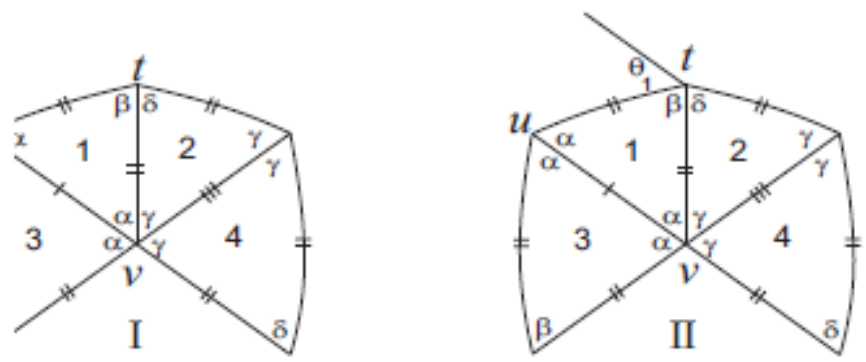

Figure 10:Local configurations near an arbitrary type III a) adjacency.

Proposition 2.4 There is a discrete family of dihedral $f$-tilings with an adjacency of type III a), and $\alpha \neq \gamma$; and there are no other such tilings. We denote the family by $\mathrm{F}^{t}, t \geq 4$. For each $t \geq 4, \mathrm{~F}^{t}$ is composed by vertices of valency four, six and $2 t$, where (both) sums of alternate angles are respectively of the form $\delta+\beta=\pi, 2 \alpha+\gamma=\pi$ and $t \gamma=\pi$.

Proof. Case A. Let us suppose that $\delta=\frac{\pi}{2}$. Then, $\frac{\pi}{4}<\gamma<\alpha<\frac{\pi}{2}$ and $\beta<\frac{\pi}{2}=\delta$.

Starting from the configuration illustrated in Figure 11, in order for the tiling to actually use both species of tile, vertex $t$ must be:

of valency six, with one of the sums of alternate angles of the form $2 \gamma+\alpha=\pi$; or

of valency $2(2+k), k \geq 1$ with one sum of alternate angles satisfying $2 \gamma+k \beta=\pi$; or

of valency $2(2+1+p), p \geq 1$, satisfying $2 \gamma+\alpha+p \beta=\pi$.

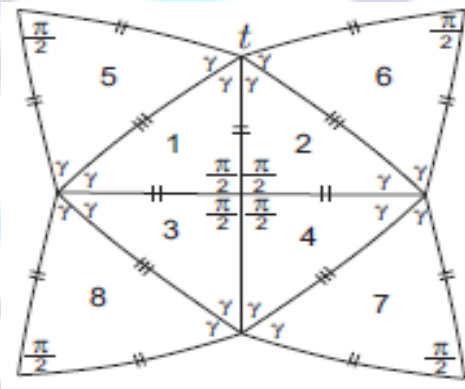

Figure 11:Local configuration for case A.

Subcase A.1 If vertex $t$ is of valency six (see Figure 12-I), then vertex $v$ must be of valency greater than four (otherwise $\beta=\frac{\pi}{2}$ ). Moreover, as $\frac{\pi}{2}+\alpha+\lambda>\pi$, for $\lambda \in\left\{\alpha, \beta, \gamma, \frac{\pi}{2}\right\}$, a set of alternate angles can only be of one of the following forms:

Subcase A.1.a $\frac{\pi}{2}+m \beta=\pi, m \geq 2$; in which case for some $m \geq 2$ then, vertex $w$ must be of valency six surrounded exclusively by angles $\alpha$ or surrounded by the sequence of angles $(\alpha, \alpha, \alpha, \alpha, \gamma, \gamma)$. However, both possibilities lead us to $\alpha=\gamma$, contradicting our hypothesis. 
Case A.1.b $\frac{\pi}{2}+\gamma+n \beta=\pi, n \geq 1$ (observe that $\frac{\pi}{2}+\gamma+\mu>\pi$, for $\mu \in\left\{\alpha, \gamma, \frac{\pi}{2}\right\}$ then vertex $w$ is partially surrounded by the angles $\alpha, \alpha, \frac{\pi}{2}$. As $2 \gamma+\frac{\pi}{2}>\pi, 2 \alpha+\beta>\pi$ and $\gamma<\alpha$, then the sum containing the alternate angles $\alpha$ and $\frac{\pi}{2}$ does not satisfy the angle folding relation.
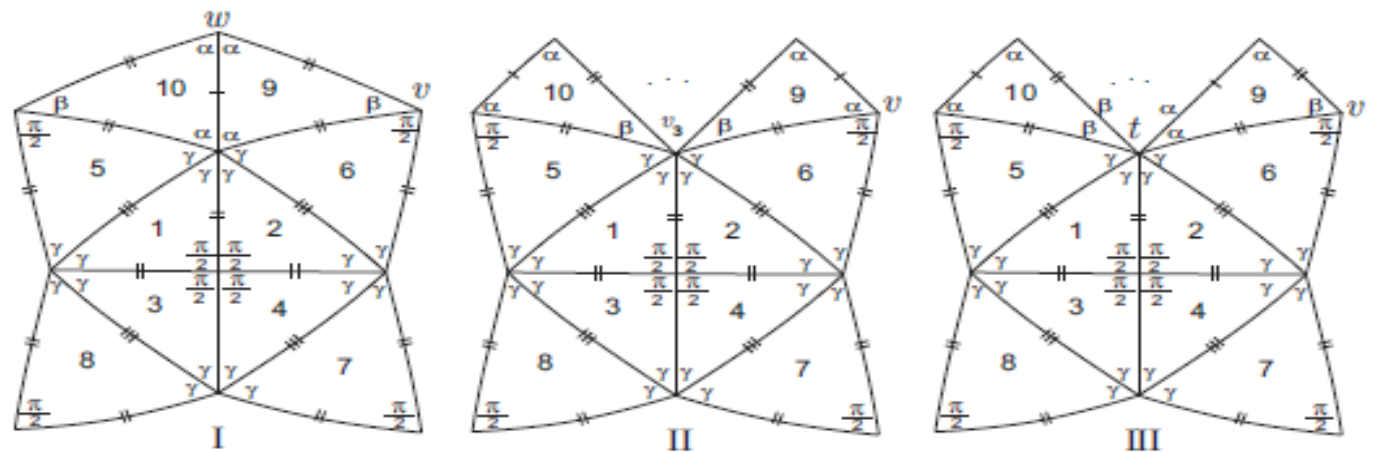

Figure 12:Local configurations for subcases A.1-3.

Subcase A.2If vertex $t$ is of valency $2(2+k)$ surrounded by the sequence of angles $(\gamma, \gamma, \gamma, \gamma, \beta, \beta, \ldots, \beta, \beta)$ (Figure 12-II), then vertex $v$ is of valency greater than four (otherwise $\alpha=\frac{\pi}{2}$ ). Nevertheless, for the same reason as above, the configuration cannot be extended.

Subcase A.3 If vertex $t$ satisfies $2 \gamma+\alpha+p \beta=\pi$, for some $p \geq 1$, then in order to avoid the situation described before, tile 9 is as shown in Figure 12-III. But again, vertex $v$ leads us to the same impossibilities as in Figure 12-I.

Case B.Assuming that $\beta=\frac{\pi}{2}$, then $\gamma<\alpha<\frac{\pi}{2}=\beta<\delta$.

The vertex surrounded partially by the angles $\beta$ and $\delta$ (in Figure 10-l) is not of valency four (otherwise, it we would have $\beta=\delta$, contrary to our assumption.) However, recalling that $2 \gamma+\delta>\pi$ and taking into account the order relation between the angles, we conclude that the sum containing the alternate angles $\delta$ and $\theta_{1}$ (Figure 10-II) cannot satisfy the angle folding relation for any choice of $\theta_{1}$.

Case C.Suppose $\delta>\beta>\frac{\pi}{2}$; then, as $\gamma<\alpha<\frac{\pi}{2}$, we have $\gamma<\alpha<\frac{\pi}{2}<\beta<\delta$. Consequently in Figure 10-II, $\theta_{1}$ must be $\alpha$ or $\gamma$. However, if the vertex is of valency four, the remaining edge is of length $b$ or $d$, and the two small angles match; this and the angle folding condition imply $\beta=\delta$, contrary to hypothesis. If the valency is greater than four, the set of alternate angles that contains $\delta$ contains at least two more angles, each at least as large as $\gamma$, and their sum would be greater than $\pi$.

Case D.Suppose that $\beta<\frac{\pi}{2}<\delta$. Then $\frac{\pi}{2}>\alpha>\gamma$, so $\delta$ is the only obtuse angle. Proposition 2.2 rules out $\gamma$ as the other angle; so the tetravalent vertices must be of the form $(\delta, \delta, \beta, \beta)$, or $(\delta, \delta, \alpha, \alpha)$. We will consider these subcases one at a time.

Subcase D.1: $(\delta, \delta, \beta, \beta)$ : We consider what the angle $\theta_{1}$ at $t$ in Figure 10-Il could be.

If we had $\theta_{1}=\alpha$, we would have $\delta+\alpha<\pi$, so the alternating angle sum needs at least one more element. But $\delta+2 \alpha>\beta+2 \alpha>\pi$, while $\delta+\alpha+\gamma>\delta+2 \gamma>\pi$; and clearly $2 \delta+\alpha$ and $\delta+\alpha+\beta$ are also greater than $\pi$. Thus $\theta_{1}=\alpha$ is incompatible with the angle folding relation. Very similar arguments rule out $\theta_{1}=\gamma$. The angle 
folding relation rules out $\theta_{1}=\delta$; so we conclude that it can only be $\beta$. We thus have, matching bases, the configuration of Figure 13.

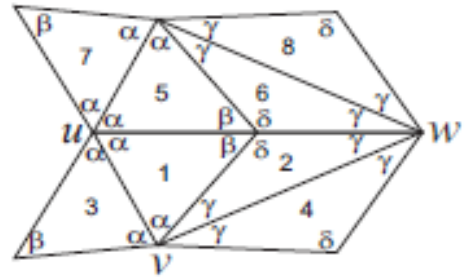

Figure 13: Local configuration for subcase D.1.

Consider the vertex at $u$, where an alternate-angle sum contains at least two $\alpha$ angles. As $\alpha$ is acute, there must be more angles present. The sum cannot contain a $\beta$ angle, as $2 \alpha+\delta>2 \alpha+\beta>\pi$. It cannot contain more than three $\alpha$ angles, as $2 \alpha>\pi-\beta=\delta>\frac{\pi}{2}$. Thus it must be of the form $3 \alpha=\pi, 2 \alpha+k \gamma=\pi(k \geq 1) ;$ or $3 \alpha+k \gamma=\pi$ $(k \geq 1)$. We will consider each of these options in turn.

Subcase D.1.a:Assume that $\alpha=\frac{\pi}{3}$. Since $2 \alpha+\beta>\pi$, then we get $\beta>\frac{\pi}{3}$. On the other hand, as $2 \gamma+\delta>\pi$, then $\gamma>\frac{\pi}{6}$. Therefore,

$$
\frac{\pi}{6}<\gamma<\frac{\pi}{3}=\alpha<\beta<\frac{\pi}{2}<\delta .
$$

The vertex $v$ (Figure 13) surrounded partially by the angles $\alpha, \alpha, \gamma, \gamma$ has alternate angle sums containing at least one $\alpha$ angle and one $\gamma$ angle. From the equality and inequalities in (13) we deduce that:

$$
2 \alpha+\gamma, \alpha+2 \gamma<\pi<\alpha+\gamma+\delta, 2 \alpha+2 \gamma, \alpha+2 \beta+\gamma, \alpha+\beta+2 \gamma, \alpha+4 \gamma
$$

We conclude that the alternate angle sums can only be of the form $\alpha+\gamma+\beta=\pi$ or $\alpha+3 \gamma=\pi$. As these have different numbers of angles, they cannot coexist at the same vertex. We consider the two subcases:

Subcase D.1.a.i: $\alpha+\gamma+\beta=\pi$. In this case we may add some new cells to the configuration in Figure 10-Il and a vertex $x$ surrounded partially by two adjacent angles $\alpha$ and one angle $\delta$ arises; see Figure 14-I. But then one set of alternate angles contains $\alpha$ and $\delta$; but this is impossible, as $\alpha+\delta<\pi<\alpha+\delta+\lambda$ for any $\lambda \in\{\alpha, \beta, \gamma, \delta\}$.

Subcase D.1.a.ii: $\alpha+3 \gamma=\pi$. Tiles 9-23 of (Figure 14-II) are forced, yielding the configuration shown, in which a vertex $y$ is surrounded by three angles $\delta$, violating the angle folding relation.

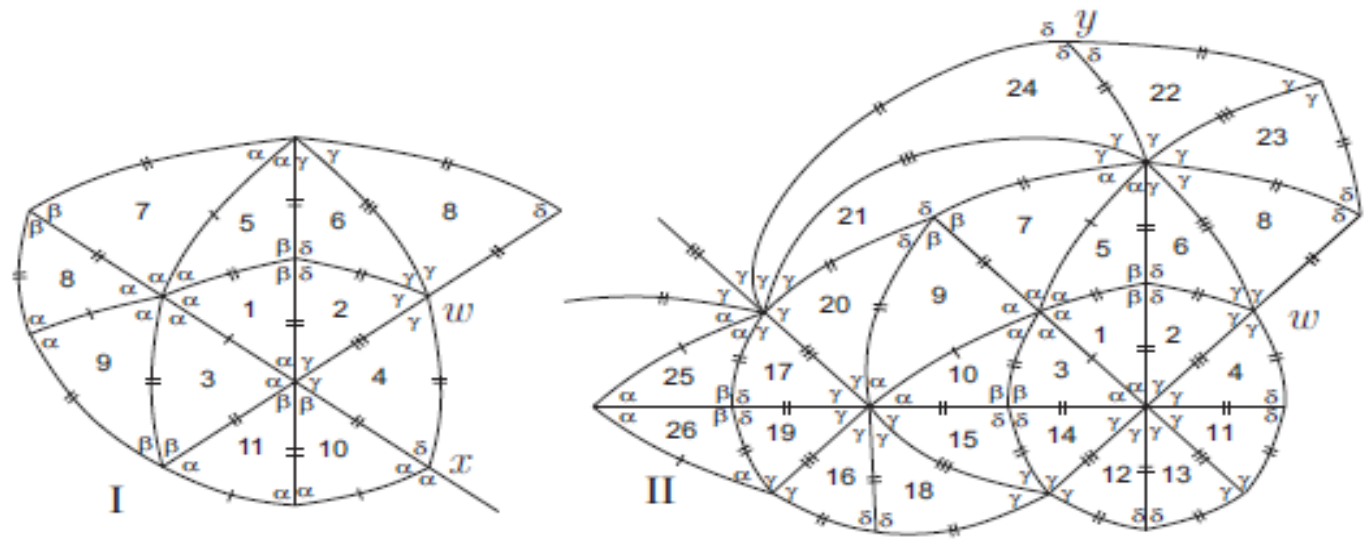

Figure 14:Local configurations for subcase D.1.a. 
Subcase D.1.bAssume that $2 \alpha+k \gamma=\pi$, for some $k \geq 1$. We have $2 \gamma+\delta>\pi$ and $\delta+\beta=\pi$, so $\beta<2 \gamma$ . Then if $k>1$, we would have

$$
\pi=2 \alpha+k \gamma \geq 2 \alpha+2 \gamma>2 \alpha+\beta>\pi .
$$

As this is impossible, $k=1$ : that is, $2 \alpha+\gamma=\pi$.

The vertex $w$ in Figure 13, partially surrounded by four consecutive angles $\gamma$, has an alternate angle sum including at least two $\gamma$ angles. The options are $m \gamma=\pi, 2 \gamma+n \beta=\pi(n \geq 1), 3 \gamma+n \beta=\pi \quad(n \geq 1)$, or $m \gamma+\alpha=\pi$. Again, we consider these subcases in order.

Subcase D.1.b.i:Suppose that a set of alternate angles at $w$ has $m \gamma$ angles (so $m \gamma=\pi$.) For $m=3$, the triangles $T_{1}$ and $T_{2}$ would be congruent, so $m \geq 4$. In this case, the configuration shown in Figure 13 extends to a complete $f$-tiling; all tiles are forced, for instance in the order shown in Figure 15. It has $8 m$ triangular faces, equally distributed between the two congruence classes, and we have three types of vertices: valency four, six and $2 m$ whose both sums of alternate angles satisfy respectively, $\delta+\beta=\pi, 2 \alpha+\gamma=\pi$ and $t \gamma=\pi$. The next figures show 2D and $3 \mathrm{D}$ representations of $\mathrm{F}^{4}$. This family of tilings maybe obtained from the family of dihedral $f$-tilings ${ }^{L} \mathrm{R}^{m}, m \geq 4$, with an isosceles triangle and a non-equiangular rhombus as prototiles, described in [7], by subdividing the rhombs.
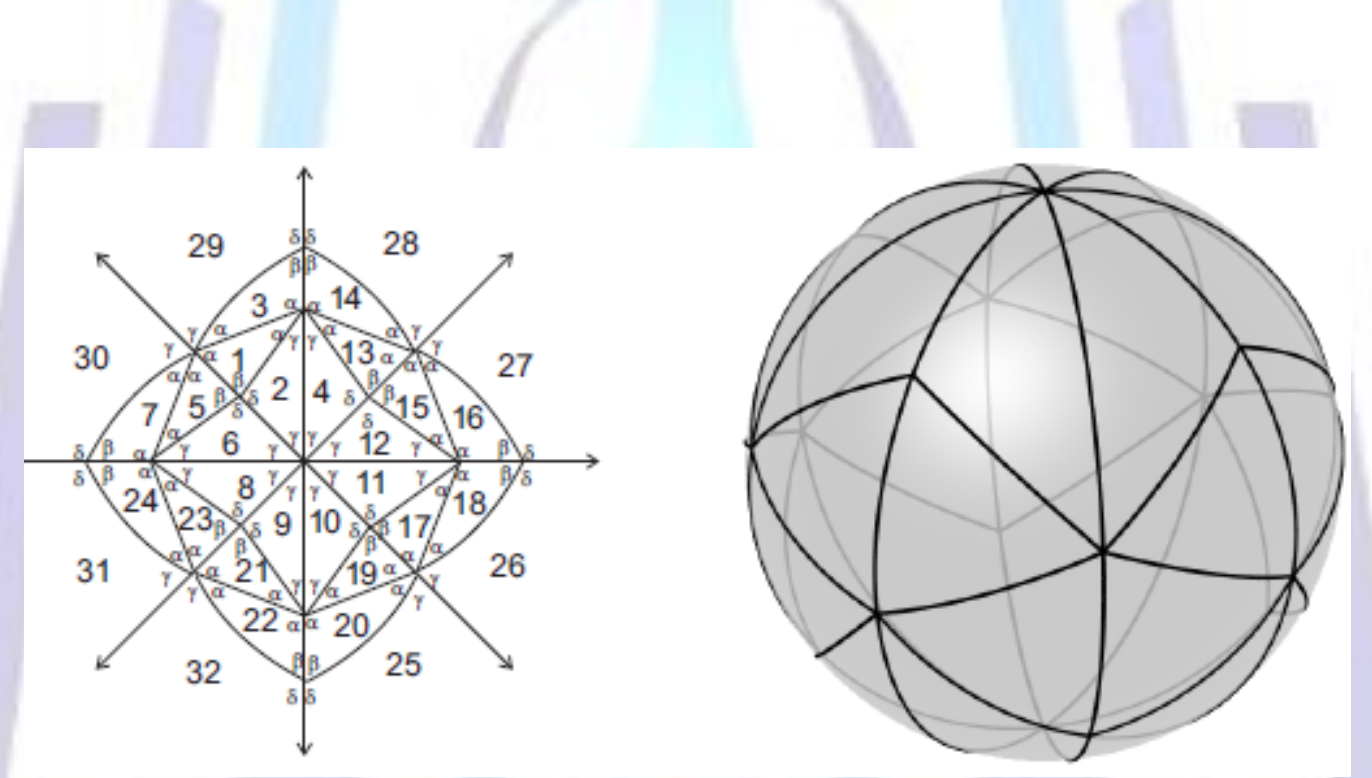

Figure $15: 2 \mathrm{D}$ and $3 \mathrm{D}$ representation of $\mathrm{F}^{4}$.

Subcase D.1.b.ii:Suppose $w$ has a set of alternate angles with two $\gamma$ angles and $n \beta$ angles (so $2 \gamma+n \beta=\pi$ .) The configuration in Figure 10-II extends to the one illustrated in Figure 16-I. The vertex $x$ surrounded partially by the consecutive angles $\delta, \alpha, \alpha$ is not of valency four, since $\beta \neq \alpha$. However, $\delta+2 \alpha>\delta+2 \beta>\pi$, $\delta+\alpha+\gamma>\delta+2 \gamma>\pi$, while $2 \delta+\alpha>\delta+\alpha+\beta>\pi$. Thus no set of alternate angles containing $\delta$ and $\alpha$ can satisfy the angle folding condition.

Subcase D.1.b.iii:Suppose $w$ has a set of alternate angles with three $\gamma$ angles and $n \beta$ angles (so $3 \gamma+n \beta=\pi$.) Then tile 11 must be a $T_{2}$, positioned as shown in Figure 16-II, to avoid the just-examined impossible configuration at $x$. But there must be $T_{1}$ triangles with $\beta$ angles at $w$; as these cannot match an edge of length $d$, one must be positioned as triangle 12. But then the same $\delta, \alpha, \alpha$ configuration arises at $y$.

Subcase D.1.b.iv:Suppose $w$ has a set of alternate angles with $m \gamma$ angles and an $\alpha$ angle (so $n \gamma+\alpha=\pi$.) Completing $v$, we arrive at the configuration of Figure 16-III, where vertex $z$ is surrounded partially by the consecutive angles $\delta, \beta, \delta$. As $2 \delta>\pi$, this cannot occur in an $f$-tiling.

Subcase D.1.C:Suppose now that $3 \alpha+q \gamma=\pi, q \geq 1$. If $q \geq 2$, we would have 


$$
2 \alpha+\beta<3 \alpha+\beta<\pi-q \gamma+2 \gamma=\pi+(2-q) \gamma \leq \pi .
$$

But this is impossible; so $q=1$ and $3 \alpha+\gamma=\pi$. Now, recall that $\delta+2 \gamma>\pi=\delta+\beta$; so $2 \gamma>\beta$. But then

$$
3 \alpha+\gamma=\pi<2 \alpha+\beta<2 \alpha+2 \gamma
$$

contradicting our assumption that $\alpha>\gamma$.
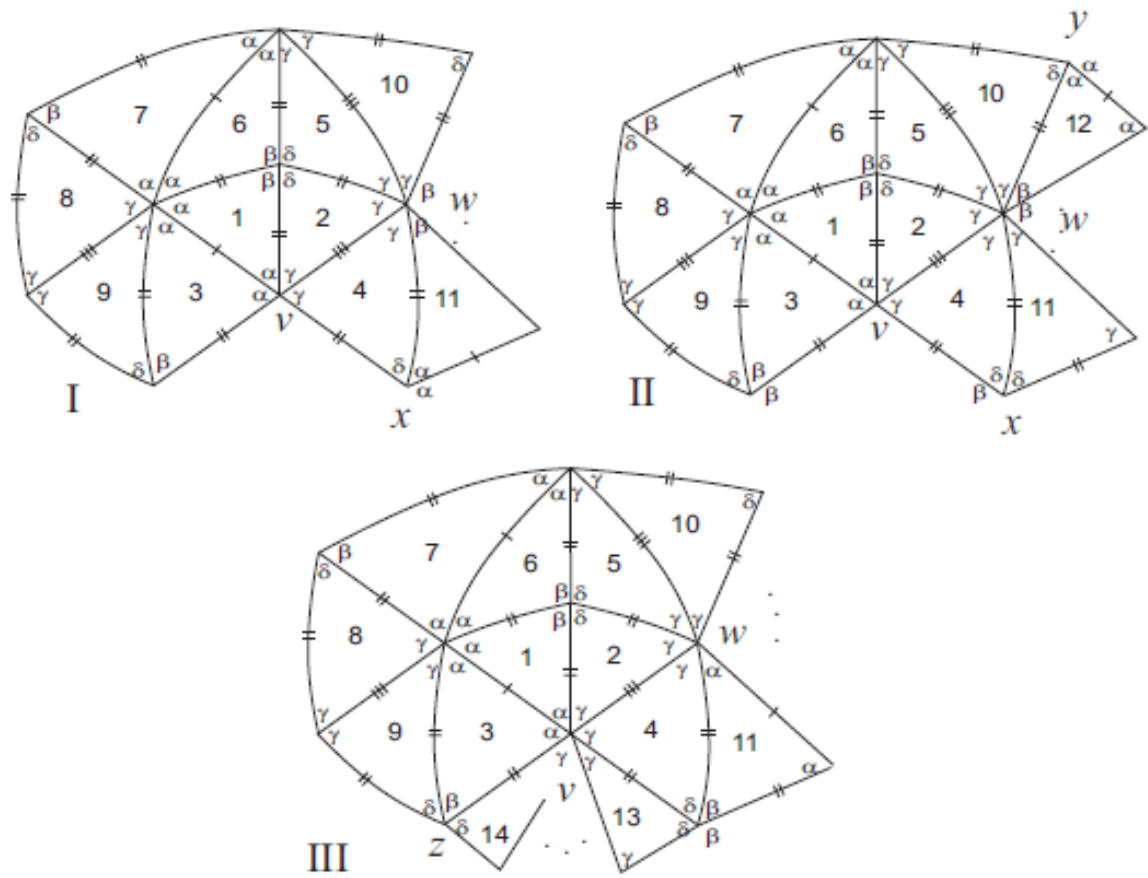

Figure 16:Local configurations for subcases of D.1.b.

Subcase D.2:Suppose that there exist vertices of valency four surrounded by the angles $\delta, \delta, \alpha, \alpha$. These cannot be the only vertices with $\delta$ angles (see Figure 10.) But the vertex $x$ cannot be tetravalent; and any set of three or more angles containing $\{\delta, \delta\},\{\delta, \alpha\}$, or $\{\delta, \gamma, \gamma\}$ must sum to more than $\pi$. We conclude that if the vertex $x$ can be completed in accordance with the folding condition, the alternate angle sum has the form $\delta+m \beta=\pi$ for $m \geq 2$ or $\delta+m \beta+\gamma=\pi$ for $m \geq 1$. In either case, $\beta<\gamma$ and $\beta<\alpha$. From the latter, and the fact that $2 \alpha+\beta$ and $2 \gamma+\delta$ are both greater than $\pi$, we get

$$
\alpha>\frac{\pi}{3}, \delta<\frac{2 \pi}{3}, \text { and } \gamma>\frac{\pi}{6} .
$$

Clearly $\alpha+\delta=\pi$; combining this with the inequality $2 \alpha+\beta>\pi$, we get

$$
\alpha+\beta>\delta \text {. }
$$

These inequalities will be used below.

Subcase D.2.a:Suppose that, at $t$, we have $\theta_{1}=\beta$ (Figure 17). Then there are three consecutive $\alpha$ angles at vertex $u$, and a set of alternate angles that contains two $\alpha$ angles. As $\alpha<\frac{\pi}{2}$, at least one more angle is needed; but, as $2 \alpha+\gamma>2 \alpha+\beta>\pi$, the folding condition is violated. 


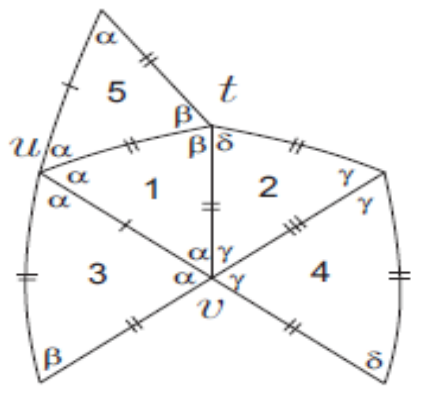

Figure 17:Local configurations for subcase D.2.a

Subcase D.2.b:We must, therefore, have $\theta_{1}=\gamma$, and $\delta+\gamma<\pi<\delta+2 \gamma, \delta+\alpha+\gamma$; so the alternate angle sum at $t$ containing $\delta$ and $\gamma$ can only be

$$
\delta+m \beta+\gamma=\pi \text { for } m \geq 1 .
$$

Consequently $\beta<\gamma<\alpha<\frac{\pi}{2}<\delta$. Then triangles 3-12 are forced, in the order shown.(5 is forced because $\theta_{1}=\gamma ; 9$ is forced because of the necessity for $\beta$ angles; and the others are obvious.)

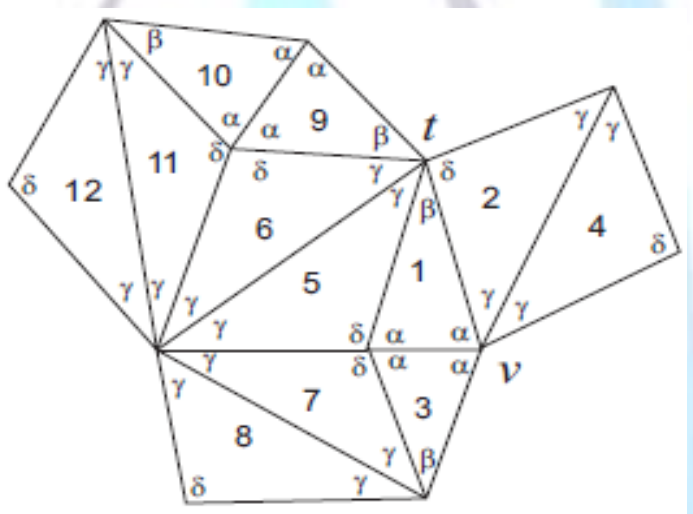

Figure 18: Local configurations for subcase D.2.b

Now, the vertex $v$, surrounded partially by angles $\alpha, \alpha, \gamma, \gamma$, cannot have $\delta$ angles. Suppose there are more than two $\alpha$ angles at $v$; then $2 \alpha+\gamma \leq \pi$. But $2 \gamma>\alpha$, so $\alpha<\frac{2 \pi}{5}$. Then we have $\beta>\frac{\pi}{5}$, and $\beta+\gamma+\delta>\alpha+\delta=\pi$, making it impossible for the folding criterion to be satisfied at $t$. We conclude that there can be only two $\alpha$ angles at $v$.

If $\alpha+q \gamma=\pi$, by (2.2) we have $q<4$. Furthermore,

$$
\alpha+\beta+2 \gamma>2 \alpha+\beta>\pi
$$

so we cannot have $\alpha+p \beta+q \gamma$ with $p \geq 1$ unless $q=1$. It follows that the sums of alternate angles at $v$ must be of the form $\alpha+2 \gamma=\pi, \alpha+3 \gamma=\pi$, or $\alpha+p \beta+\gamma=\pi$. We will study each of these three subcases.

Subcase D.2.b.i:Suppose the sum to be $\alpha+2 \gamma=\pi$. In this, and the next subcase, triangle 13 is forced at $v$. There is now a partial set of alternate angles at $S$ containing a $\delta$ and a $\gamma$; as at $t$, triangle 14 is forced (see Figure 19).

Triangles 15-17 follow immediately. We now have a vertex with eight $\gamma$ angles, so $4 \gamma \leq \pi$. But this makes $\alpha \geq \frac{\pi}{2}$, which is impossible. 


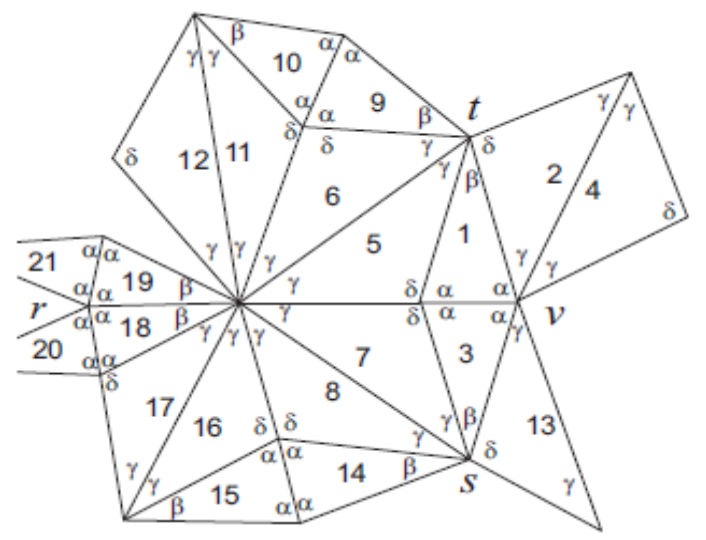

Figure 19:Local configuration for subcases D.2.b.i and D.2.b.ii

Subcase D.2.b.ii:Suppose the sum to be $\alpha+3 \gamma=\pi$. We obtain triangles $13-17$ as in the previous subcase. We note that triangles 12 and 17 cannot have an edge in common, as this would imply $4 \gamma=\pi$, whence $\gamma=\alpha$. Therefore, triangles $5-8,11,12,16$, and 17 do not fully surround the vertex on their own. But $3 \gamma=\pi-\alpha=\delta$, so $5 \gamma=\delta+2 \gamma>\pi$; it follows that all remaining angles must be $\beta$ angles. The folding condition requires there to be at least two $\beta$ angles at this vertex. Triangles 18-21 must therefore be as shown; but this creates a vertex $r$ with four $\alpha$ angles, and this cannot be completed in accordance with the folding condition.

Subcase D.2.b.iii:Suppose the sum to be $\alpha+p \beta+\gamma=\pi, m \geq 1$. Combining this with (2.4), we have $\alpha+p \beta=\delta+m \beta$; and as $\alpha<\delta$, we have $m>p$.

On the other hand, substituting (2.3) into (2.4) gives us the inequality

$$
\alpha+(m+1) \beta+\gamma>\pi \text {. }
$$

We conclude that $m+1>p>m$, which is clearly impossible as both $m$ and $p$ are natural numbers.

\subsection{Tilings in which adjacency of type III a) is absent.}

All tilings with one or more adjacencies of type III a) have now been classified. Any remaining tiling must have only adjacencies of type III b), so that whenever noncongruent triangles share a common edge, they "point the opposite way".

Proposition 2.5 A spherical $f$-tiling with $\alpha=\gamma=\frac{\pi}{2}$, which is without adjacencies of type III a), is of the form $\mathrm{B}^{p, 0,0 q}$ (as defined in Proposition 2.3) for some $p>q>0$. For given $p$ and $q$ there is only one tiling in this class (see Figure 21-III).

Proof. Assume that $\alpha=\gamma=\frac{\pi}{2}$. We assume a type III b) adjacency (Triangles 1 and 2 of Figure 20.) Triangles 3 and 4 are forced by pairing sides of length $a$ and $d$. The vertex $v_{1}$ cannot be tetravalent, and thus cannot have a third right angle. If there were any $\delta$ angle there, some $\delta$ angle would be adjacent to a $\beta$ angle, creating a forbidden type III a) adjacency. We conclude that the fan at $v_{1}$ consists entirely of $\beta$ angles (Figure 20-II.) Pairing edges of length $b$ gives the same structure at $v_{3}$, while $v_{2}$ and $v_{4}$ consist entirely of $\delta$ angles (Figure 20-III.) We thus have $2 p \beta=2 q \delta=\pi$ , and (if we take $\beta<\delta$ ), $p>q>1$. 

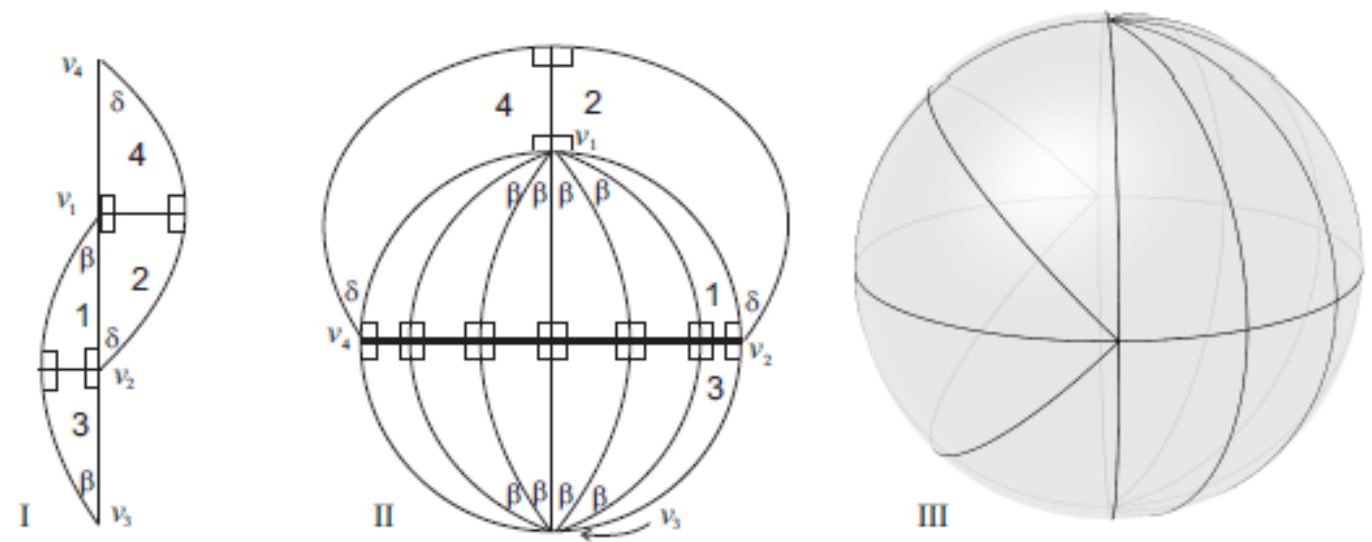

Figure 20:Local and global configuration with $\alpha=\gamma=\frac{\pi}{2}$

Otherwise, without loss of generality we have $\gamma<\alpha, \beta<\delta$.

Lemma 2.1 If $T_{1}$ and $T_{2}$ yield an $f$-tiling with adjacency of type III b), and $\gamma$ and $\alpha$ are not right, then $\gamma<\alpha<\frac{\pi}{2}<\delta$.

Proof.We have triangles 1,2,3, and 4 as in Figure 21-I. Suppose $\alpha>\frac{\pi}{2}$; then by equation $1.2 \gamma>\frac{\pi}{2}$ also. But then, however we cover the edge $\overline{v_{1} v_{2}}$, we obtain a third obtuse angle at $v_{1}$ or $v_{2}$, forbidden by the folding relation; so we must have $\alpha<\frac{\pi}{2}$. The existence of vertices of valency four requires that $\delta>\frac{\pi}{2}$.

Lemma 2.2 In an $f$-tiling without adjacencies of type III a):

$\{\beta, \delta\}$

1. a vertex with at least one $\alpha$ angle and at least one $\gamma$ angle must also have at least two angles from

2. a vertex with at least one $\beta$ angle and at least one $\delta$ angle must have at least two adjacent pairs of base angles.

Proof.If ( e.g.) both $\alpha$ angles and $\gamma$ angles exist, they must be isolated from each other to prevent Type III a) adjacencies. To do this in a cycle requires two other angles. Furthermore, $\alpha$ angles always occur in adjacent pairs, as do $\gamma$ angles.

The next lemma shows that Lemma 2.2(ii) is vacuous.

Lemma 2.3 If $T_{1}$ and $T_{2}$ yield an $f$-tiling without adjacency of type III a), and $\gamma<\alpha<\frac{\pi}{2}$, then:

1. all tetravalent vertices are of the form $(\delta, \delta, \alpha, \alpha)$;

2. $\alpha+\delta=\pi$;

3. $\alpha<2 \gamma$;

4. any vertex with a $\delta$ angle is of the form $(\delta, \delta, \alpha, \alpha)$.

Proof.By Proposition 2.1 there are tetravalent vertices, with supplementary angles; one of these must be obtuse, hence (by Lemma 2.1) $\delta$ or (possibly) $\beta$. Lemma 2.2 forbids $(\delta, \delta, \beta, \beta)$. Proposition 2.2 rules out $(\delta, \delta, \gamma, \gamma)$ and $(\alpha, \alpha, \beta, \beta)$. Suppose we have a tetravalent vertex with angles $(\beta, \beta, \gamma, \gamma)$. Then triangle 5 must be as shown in Figure 21-II, and at the vertex $w$ a set of alternate angles contains $\delta$ and $\alpha$. But as $\delta+\alpha>\beta+\gamma=\pi$, this violates 
the folding condition. The first part of the lemma follows by elimination; the second and third parts are immediate corollaries.

Suppose that a $\delta$ and $\beta$ angle are found at the same vertex. By the previous lemma, they must be separated on both sides by pairs of base angles; and as $\alpha+\delta=\pi$, neither pair can consist of $\alpha$ angles. The vertex thus has two pairs of $\gamma$ angles sharing a long edge (Figure 21-III.) But then one of the sums of alternate angles contains, at least in part, $\delta+2 \gamma$, which is impossible.
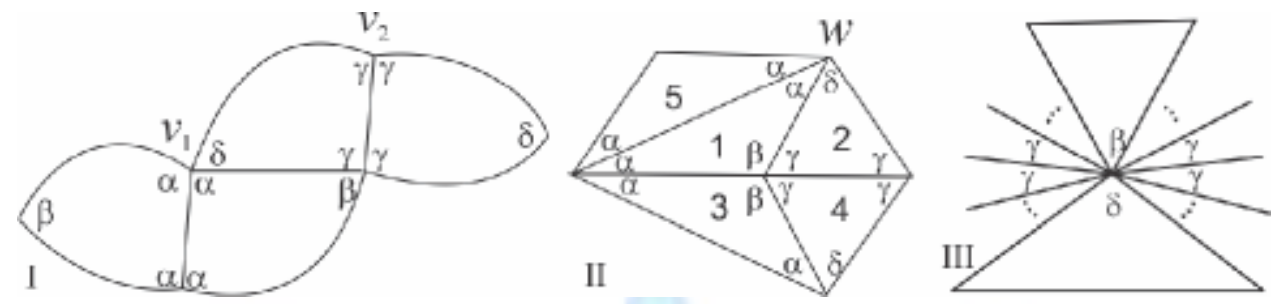

Figure 21: Local configurations from lemmas 2.1 and 2.3

Lemma 2.4 In an $f$-tiling without adjacency of type III a), in which the base angles are not right, every adjacent pair of noncongruent triangles extends to the local configuration of figure 22.

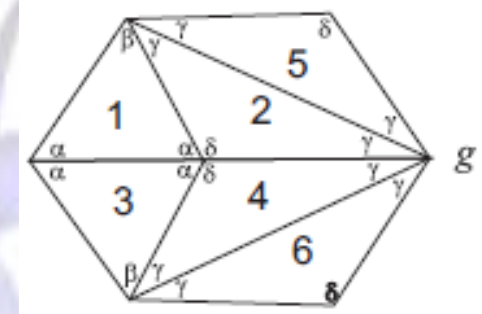

Figure 22:Configuration common to all $f$-tilings without type III a) adjacency in which $\alpha, \gamma$ are not right.

Proof.Let triangles 1 and 2 be noncongruent; then they have type III b) adjacency (Figure 22). Then (Lemma 2.3) triangles 3 and 4 must be as shown, and base matching forces triangles 5 and 6 .

The next lemma limits our choices for the vertex labelled $g$ in Figure 22.

Lemma 2.5 In an $f$-tiling without adjacency of type III a), in which the base angles are not right, a vertex with four adjacent $\gamma$ angles has no $\delta$ or $\alpha$ angles.

Proof.The vertex cannot have a $\delta$ angle by Lemma 2.3(4). If it has an $\alpha$ angle, it has at least two adjacent $\alpha$ angles, as the bases must be paired. But then some alternating angle sum contains two $\gamma$ angles, an $\alpha$, and a $\beta$. But by Lemma 2.3(3), $\alpha+\beta+2 \gamma>2 \alpha+\beta>\pi$, violating the folding condition.

Proposition 2.6 The $f$-tilings without adjacency of type III a), in which $\gamma$ and $\alpha$ are not right angles, are:

$$
\begin{aligned}
& \mathrm{J}^{k}, k \geq 3: \alpha+\delta=\pi, \beta+2 \gamma=\pi \text { and } \gamma=\frac{\pi}{k} \\
& \mathrm{~K}^{t}, k \geq 4: \alpha+\delta=\pi, \beta+3 \gamma=\pi \text { and } \gamma=\frac{\pi}{t} \\
& \mathrm{~L}^{k}, k \geq 3: \alpha+\delta=\pi, \beta+2 \gamma=\pi \text { and } \alpha=\frac{\pi}{k} \\
& \mathrm{M}^{k}, k \geq 3: \alpha+\delta=\pi, \beta+3 \gamma=\pi \text { and } \alpha=\frac{\pi}{k}
\end{aligned}
$$




$$
\begin{aligned}
& \mathrm{N}^{k}, k \geq 3: \alpha+\delta=\pi, \alpha+\beta+\gamma=\pi \text { and } \gamma=\frac{\pi}{k} \\
& \mathrm{P}: \alpha+\delta=\pi, 2 \beta+\gamma=\pi, \alpha=\frac{\pi}{3} \text { and } \gamma=\frac{\pi}{5} \\
& \mathrm{Q}: \alpha+\delta=\pi, 2 \beta+\gamma=\pi \text { and } \alpha=\frac{\pi}{3} .
\end{aligned}
$$

Proof.We will consider separate cases based on what nontetravalent vertices containing one or more $\alpha$ angles exist. It is possible that there are none (case A below), or that a vertex with only $\alpha$ angles exists (case B). The following lemmas help classify the remaining cases.

Lemma 2.6 In an $f$-tiling without adjacency of type III a), in which the base angles are not right and no vertex is surrounded entirely by $\alpha$ angles, no vertex has more than two $\beta$ angles.

Proof. If there are no $\alpha$-only vertices, it is impossible for two $\beta$ angles to be adjacent at a vertex $v$. Matching short bases, we find that a neighboring vertex $w$ has four adjacent $\alpha$ angles (figure 23.) They do not totally surround $w$; what can fill the gap? Lemma 2.3 rules out $\delta$ angles. As $2 \alpha+\beta>\pi, \beta$ angles cannot be used. This leaves only $\gamma$ angles, which would create a type IIIa) adjacency. (There might more than four $\alpha$ angles at $w$; the important thing is that at some point the sequence of $\alpha$ angles must end, and there is no legal choice for the next angle.)

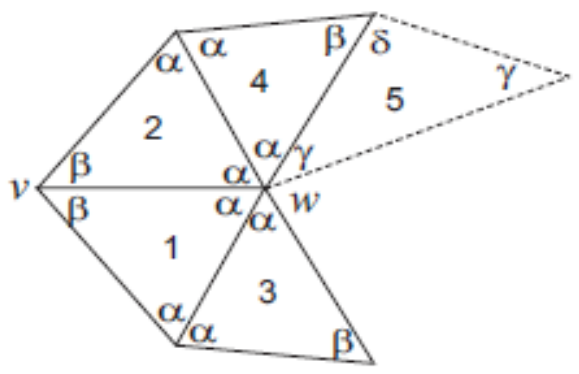

Figure 23:An impossible configuration

It follows that if $v$ had more than two $\beta$ angles, each pair (in cyclic order) would have to be separated by an $\alpha$ angle or two $\gamma$ angles. But then some alternating angle sum would have the form $a \alpha+b \beta+2 c \gamma=\pi$ for $a+c \geq b>1$. But

$$
a \alpha+b \beta+2 c \gamma \geq(a+c) \alpha+\beta \geq 2 \alpha+\beta>\pi
$$

so this is impossible.

A similar argument, in conjunction with Lemma 2.2, gives the following; details are omitted.

Lemma 2.7 In an $f$-tiling without adjacency of type III a), in which the base angles are not right, no vertex with an $\alpha$ angle has more than two $\gamma$ angles.

We conclude that the only other possibility (case $\mathrm{C}$ ) is that $\alpha+\beta+\gamma=\pi$, and some vertex has two $\alpha$ angles, two $\beta$ angles, and two $\gamma$ angles. (It is not obvious that this case and case B are disjoint; this will follow from the analysis of case B.)

\section{Case A: The angle $\alpha$ only appears at tetravalent vertices.}

In this case, let ( e.g.) $n_{\alpha}$ be the number of $\alpha$ angles in the tiling. We have $n_{\gamma}=2 n_{\delta}=2 n_{\alpha}=4 n_{\beta}$. But this implies that some individual vertex must have at least four times as many $\gamma$ angles as $\beta$ angles. As $4 \gamma+\beta>2 \alpha+\beta>\pi$, we conclude that this is only possible if there are no $\beta$ angles at this vertex. But then (Lemma 2.2) there are no $\alpha$ angles or (Lemma 2.3) $\delta$ angles there either.

The tiling thus contains a complete rosette of $2 k T_{2}$ triangles $(k \geq 3)$, as shown(figure 24-I). The $\delta$ angles at $u$ must 
be paired with $\alpha$ angles, forcing a ring of $2 k T_{1}$ triangles. These in turn create vertices $v$ with $\alpha$ angles that require $\delta$ angles to complete.

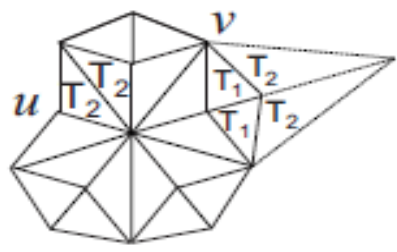

I

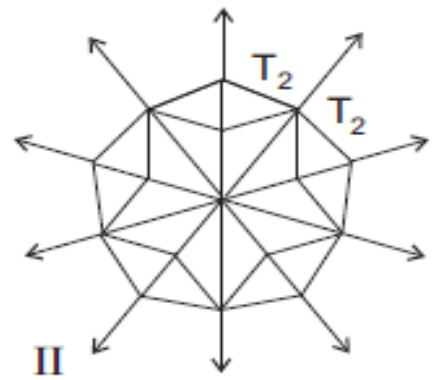

II

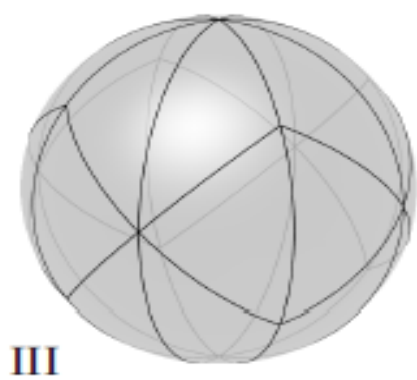

Figure 24:Case A, subcase A.1, and the tiling $\mathrm{J}^{4}$

But then the vertices $v$ have two $\beta$ angles and at least four $\gamma$ angles. By lemmas 2.6 and 2.7, any further angles must be $\gamma$. We cannot have eight or more $\gamma$ angles in total, as then the sums of alternating angles would be $\beta+4 \gamma>\beta+2 \alpha>\pi$.

Subcase A.1:There are four $\gamma$ angles at $v$, and $\beta+2 \gamma=\pi$. Filling the gap at each vertex $v$ with two more $T_{2}$ tiles completes the tiling $\mathrm{J}^{k}$ (see figure 24-II; note that the edges terminating in arrowheads all meet at the antipodal point of the original vertex.) These tilings exists for all $k \geq 3$. The 2D images show $\mathrm{J}^{5}$, the 3D image shows $\mathrm{J}^{4}$.

Subcase A.2:There are six $\gamma$ angles at $v$, and $\beta+3 \gamma=\pi$. The vertex $w$ is as shown, with $\delta$ angles that must be matched with $\alpha$ angles. The vertices $y$ now have two $\beta$ and four $\gamma$ angles and need two more $\gamma$ angles, completing the tiling $\mathrm{K}^{k}$. (Calculation shows that for $k=3$ the tile $T_{1}$ is degenerate, with $\beta=0$. The tilings $\mathrm{K}^{k}$ thus exist only for $k \geq 4$.)
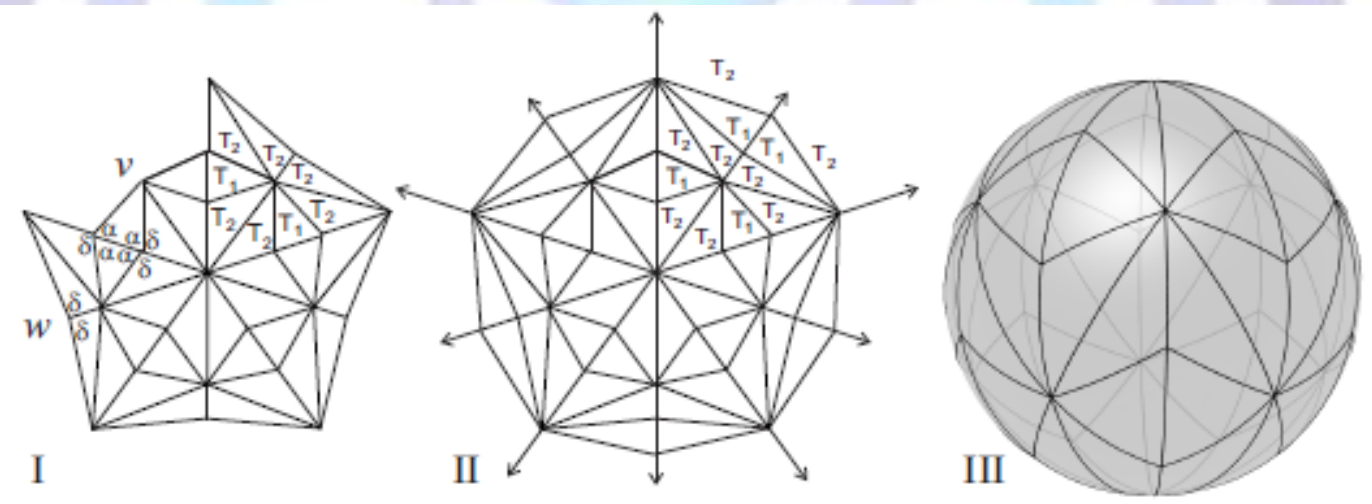

Figure 25:Subcase A.2 and the tiling $\mathrm{K}^{4}$

\section{Case B: There is at least one vertex with only $\alpha$ angles.}

Such a vertex must have $2 k \alpha$ angles for some $k \geq 3$ (see figure 26-I.) We consider one of the adjacent vertices $u$ with two $\beta$ angles, and consider what other angles can exist there.

The gap cannot be filled by two or four $\alpha$ angles, or two $\alpha$ angles and four $\gamma$ angles, because

$$
\alpha+\beta<\pi<2 \alpha+\beta<\alpha+\beta+2 \gamma .
$$

Nor can we have four more $\beta$ angles, because $\alpha \leq \pi d 3$, so $\beta>\pi d 3$ and $3 \beta>\pi$.

Similarly, we cannot have two more $\beta$ and four $\gamma$ angles, or eight $\gamma$ angles, because

$$
\pi<2 \alpha+\beta<2 \beta+2 \gamma, \beta+4 \gamma .
$$


The possible alternate-angle sums at the vertex are thus $\beta+2 \gamma=\pi$ (subcase B.1 below), $2 \beta+\gamma=\pi$ (subcase B.2), and $\beta+3 \gamma=\pi$ (subcase B.3). The first of these is clearly incompatible with either of the others. The second can only occur for $k=3$; for other values of $k, \alpha \leq \frac{\pi}{4}$, so $\beta>\frac{\pi}{2}$.
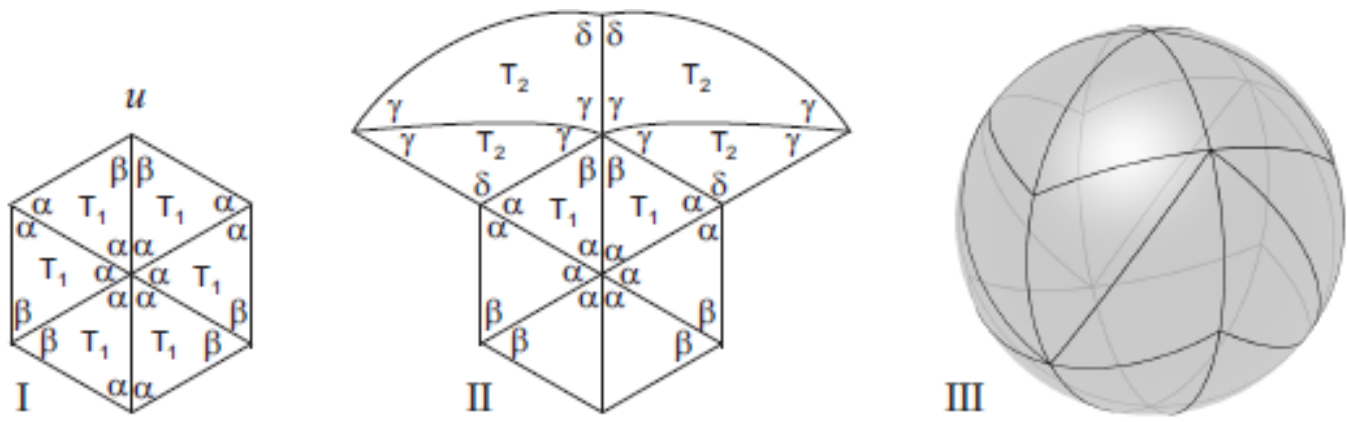

Figure 26:Case B, subcase B.1, and the tiling $\mathrm{L}^{3}$

Subcase B.1: $\beta+2 \gamma=\pi$. Each vertex $u$ with two or more $\beta$ angles must have exactly two $\beta$ angles and four $\gamma$ angles (figure 26-II.) This forces the zig-zag equatorial ring of $T_{2}$ triangles partially visible in figure 26-III. These in turn expose pairs of $\delta$ angles (figure 26-II, top), each pair requiring two more $T_{1}$ triangles by Lemma 2.3. This yields a complete tiling, $\mathrm{L}^{k}$, for each $k \geq 3$. This tiling differs from $\mathrm{F}^{k}$ in that the pairs of equatorial triangles within each lune have different orientations.

Subcase B.2: $2 \beta+\gamma=\pi$. As observed above, this can only occur when $k=3$; we then have $\alpha=\frac{\pi}{3}, \beta=\frac{\pi}{5}$, $\gamma=\frac{\pi}{10}$ and $\delta=\frac{2 \pi}{3}$. The hypothesized $\alpha$-only vertex has six $\alpha$ angles.

As always, the triangles must appear in congruent pairs with common bases; the union of two such triangles, whether $T_{1}$ or $T_{2}$, is a rhomb with angles $\frac{2 \pi}{3}$ and $\frac{\pi}{5}$. This rhomb tiles uniquely, yielding the spherical rhombic triacontahedron (the dual of the icosadodecahedron). Any tiling using the triangle tile set, then, is a subdivision of the spherical rhombic triacontahedron. The number of such subdivisions is approximately $2^{30}$ divided by 120 , the order of the icosahedral symmetry group. (This approximation treats the symmetry group of every tiling as trivial - as a result, the numerical value it gives, approximately nine million, undercounts slightly.) The estimate evaluates to around nine million but only four of these many tilings use both tiles and obey the folding condition. We will see that one has already been found, one is in a family that will be described below, and two are new sporadic tilings.

Subcase B.2.a:There is a vertex with only $\gamma$ angles. This must be surrounded by a rosette of $T_{2}$ triangles (1 in Figure 27-I) The exposed $\delta$ angles force the next ten triangles (2), all $T_{1}$. There are now exposed vertices (for instance $u$ ) with two $\beta$ and two $\gamma$ angles; we may either complete these with four more $\gamma$ angles (figure 27-II) or with two more $\beta$ angles (figure 28-I). 

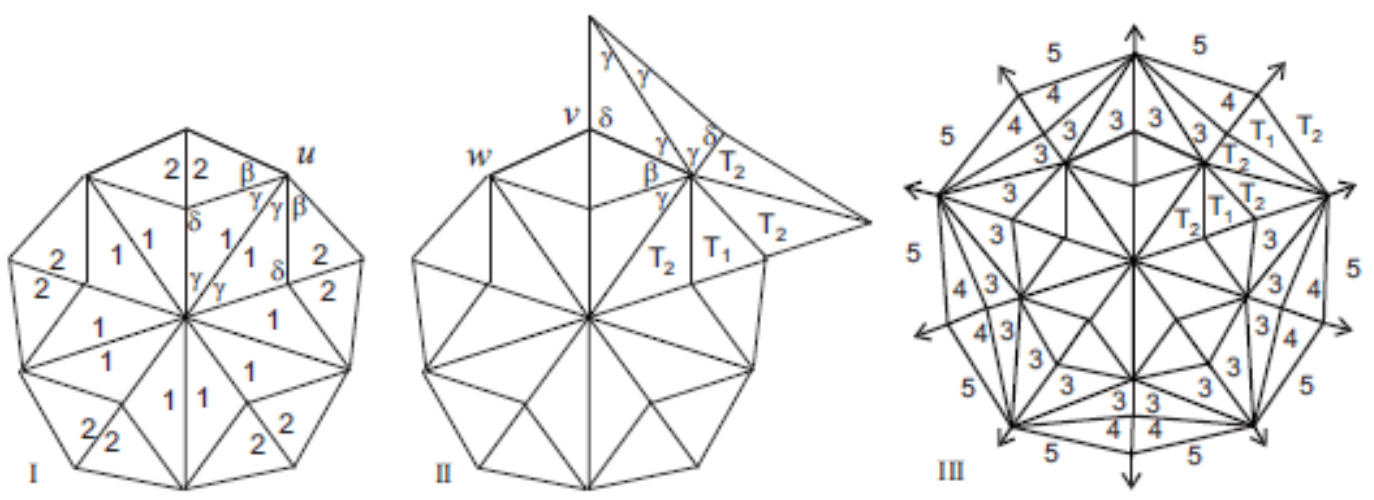

Figure 27: Subcases B.2 and B.2.a.i

Subcase B.2.a.i:The vertex $u$ has two $\beta$ angles and six $\gamma$ angles. The adjacent vertex, $v$ in figure 27-II, is tetravalent, and must be completed in accordance with Lemma 2.3. This forces a third $\gamma$ angle at $w$, and the pattern propagates around the ring of triangles labeled 3 in figure 27-III. The same lemma then forces all the triangles labelled 4 , and the final ten triangles (5) are forced at vertices that already have four $\gamma$ angles. Examining the tiling obtained, we see that while it has the correct types of tile, it is not in fact part of case B, because it does not have any vertex with only $\alpha$ angles. Rather, it is the already-identified tiling $\mathrm{K}^{5}$ (figure 25-III).

Subcase B.2.a.ii: The vertex $u$ has four $\beta$ angles and two $\gamma$ angles. The adjacent vertex ( $v$ in figure 28-I) has at least four $\alpha$ angles, and must therefore have six; again, this forces a third $\beta$ angle at the next exposed vertex, $w$, and the pattern propagates. The resulting configuration (figure 28-II) is a sporadic tiling $\mathrm{P}$ that is not part of any infinite family. Figure 28-III shows a three-dimensional view of this tiling.
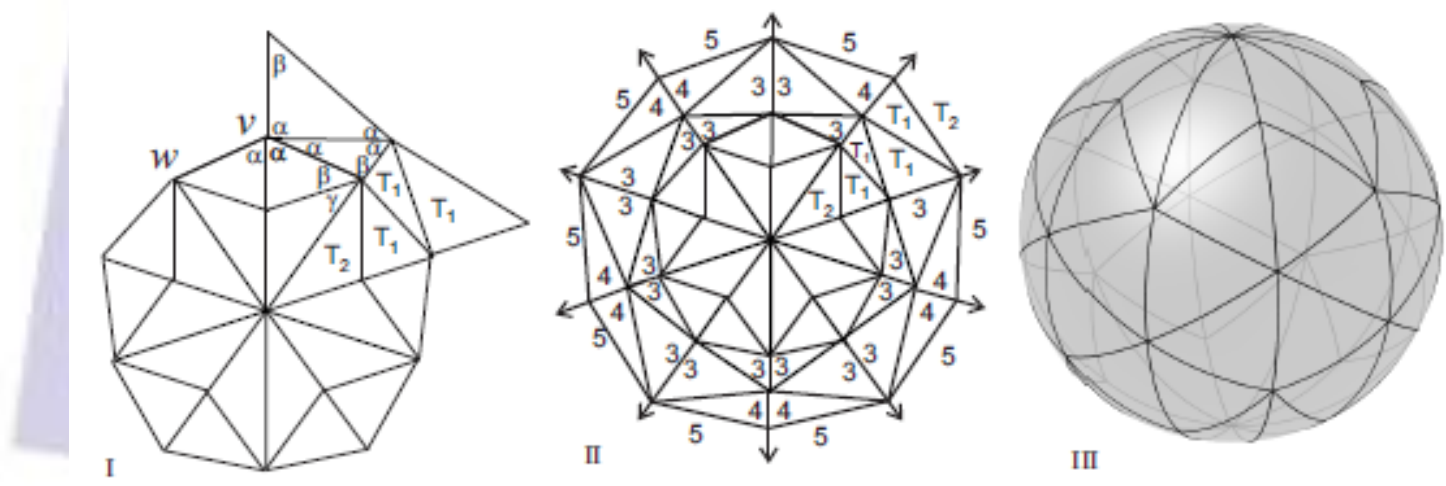

Figure 28: Subcase B.2.a.ii, and the sporadic tiling $P$

Subcase B.2.b:There is a six- $\alpha$ vertex, and no vertex with only $\gamma$ angles. We consider two subcases, depending on whether two six- $\alpha$ vertices are adjacent:

Subcase B.2.b.i:There is no vertex with only $\gamma$ angles, and there are two adjacent six- $\alpha$ vertices. By hypothesis, we have the ten $T_{1}$ triangles shown in figure 29-I. At the vertex $u$ there must be two $\gamma$ angles and a $\beta$; without loss of generality, the configuration is as in figure 29-II (the triangles labeled (3) are forced.) The vertex $v$ has six $\gamma$ angles, and by hypothesis cannot have more; and those tiles labeled (4) in figure 29-III must be as shown to avoid a vertex with only $\gamma$ angles. After this, the triangles labeled (5) are forced sequentially in clockwise order, starting at the upper right. The triangles labeled (6) in figure 29-IV are then forced, followed by those labeled (7). This completes the sporadic tiling $Q$ (figure 29-V). 


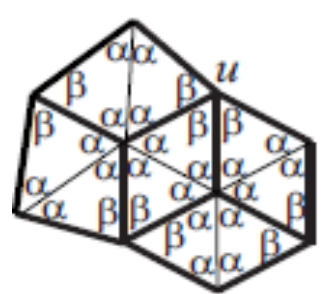

I
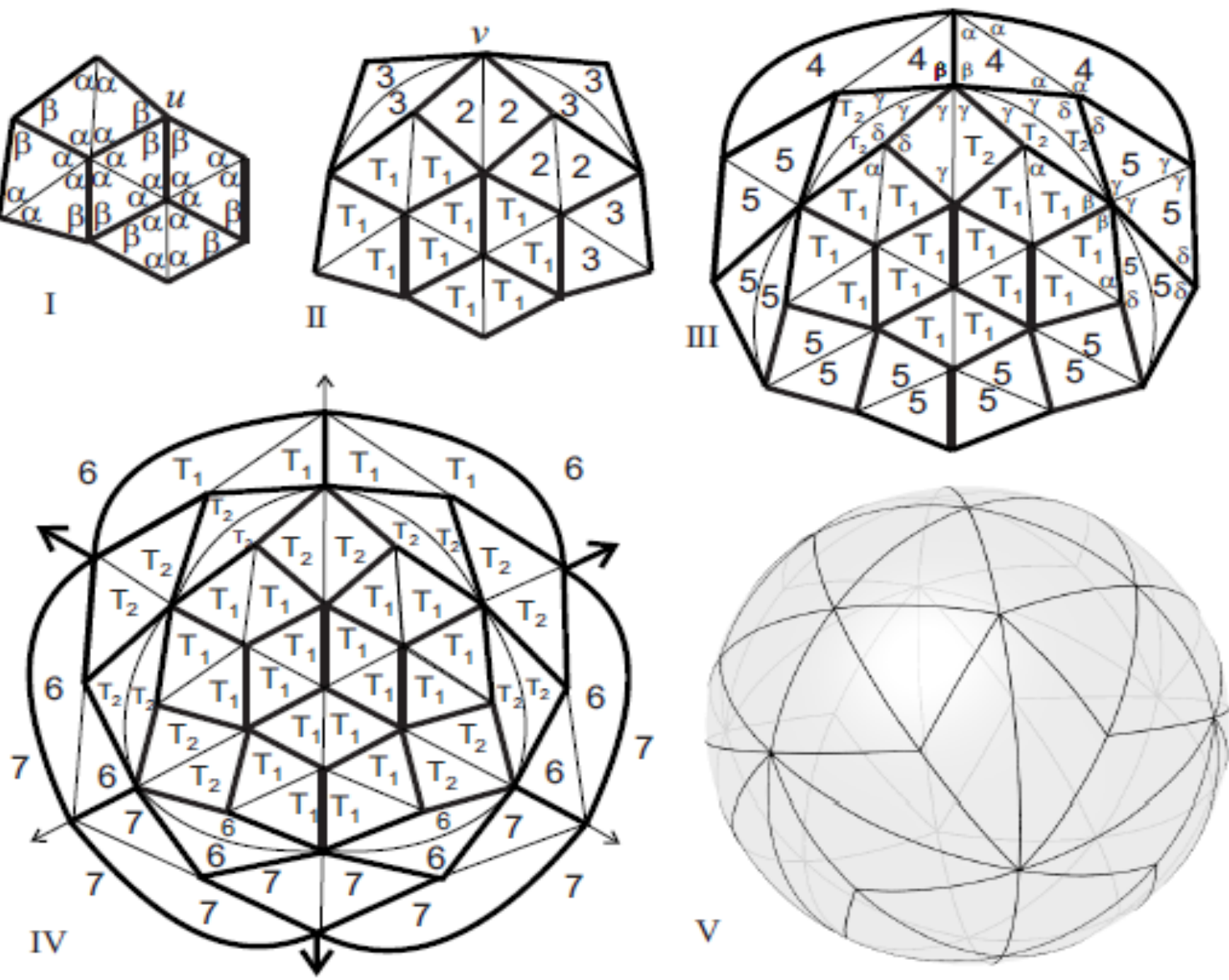

Figure 29:Subcase B.2.b.i, and the sporadic tiling $Q$

We note that in fact there is a symmetric patch of eighteen $T_{1}$ triangles at each pole, with a central hexavalent vertex surrounded by three others. The $T_{2}$ triangles form an ornate "belt" at the equator.

Subcase B.2.b.ii:There is at least one six- $\alpha$ vertex, but no two are adjacent. By hypothesis, we have a vertex surrounded by six $T_{1}$ triangles, and the three adjacent vertices with at least two $\alpha$ angles must be tetravalent (see figure 30-I.) Every other triangle in the tiling is forced, either by Lemma 2.3 or by the observation that a vertex with two $\beta$ angles and four $\gamma$ angles must be completed with two more $\gamma$ angles rather than an odd $\beta$. The resulting tiling is $\mathrm{M}^{3}$, a particular case of the $\mathrm{M}^{k}$ family described below.

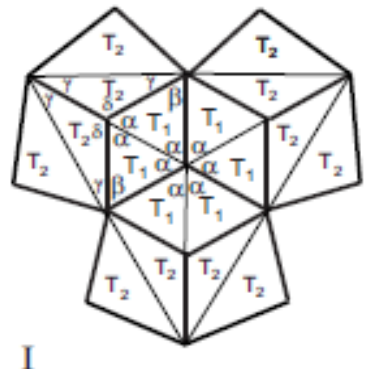

I
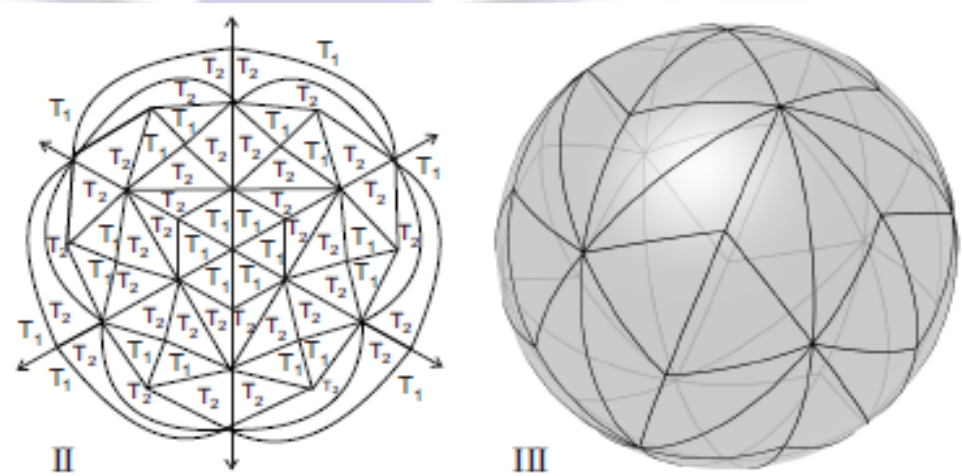

Figure 30: Subcase B.2.b.ii, and the tiling $\mathrm{M}^{3}$ 
Subcase B.3: $\beta+3 \gamma=\pi$. If $k=3$, calculation shows that we have $\alpha=\frac{\pi}{3}, \beta=\frac{2 \pi}{5}, \gamma=\frac{\pi}{5}$, and $\delta=\frac{2 \pi}{3}$. This set of angles satisfies $2 \beta+\gamma=\pi$, so any such tilings have been dealt with in subcase B.2. If $k>3$, neither $\beta+2 \gamma$ nor $2 \beta+\gamma$ is equal to $\pi$, so (as in subcase B.1) we may assume every vertex sum including a $\beta$ angle to be of the type defining the subcase, and every vertex with at least one $\beta$ angle to have exactly two $\beta$ angles and six $\gamma$ angles.

By hypothesis there is a vertex with only $2 k \alpha$ angles. Of the $2 k$ adjacent vertices, $k$ have two $\beta$ angles, and the other six triangles are thus forced. As in subcase B.2.b.ii, the rest of the tiling follows by alternately completing tetravalent vertices and vertices with two $\beta$ angles. The reader is referred to Figure 30. This tiling, $\mathrm{M}^{k}$, exists for all $k \geq 3$.
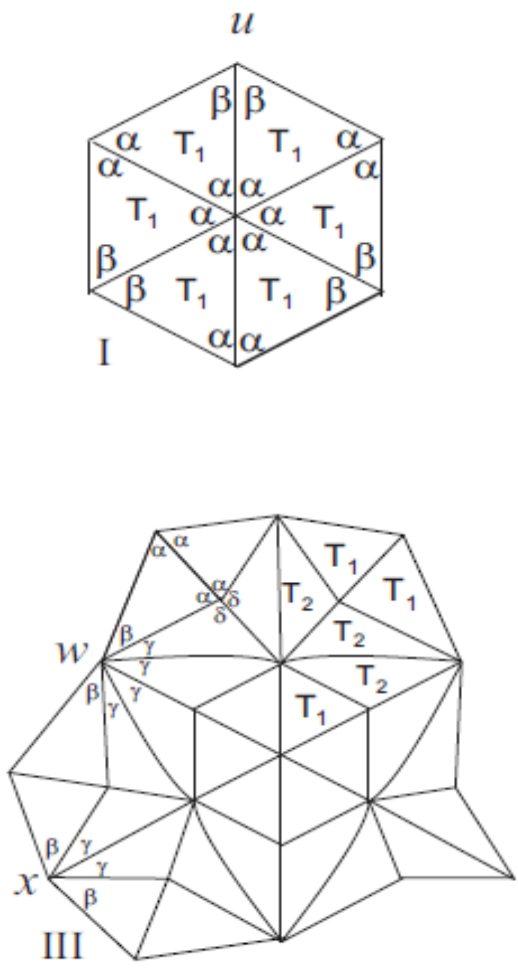
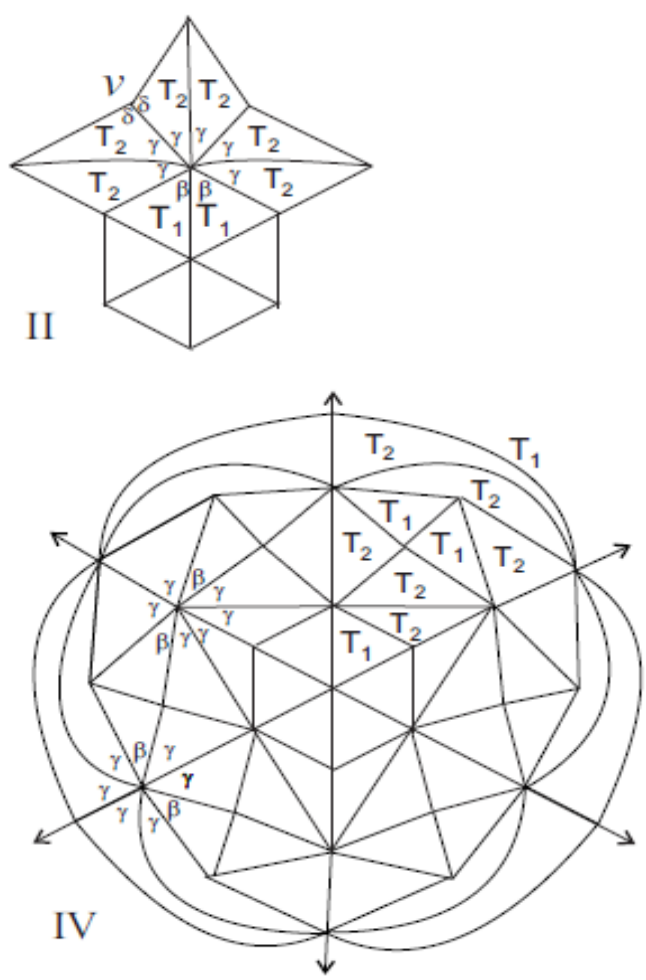

Figure 31:Subcase B.3, and the tiling $\mathrm{M}^{3}$

Case $\mathbf{C}: \alpha+\beta+\gamma=\pi$. None of the four types of "case B" tiling contains a vertex with two $\alpha$ angles, two $\beta$ angles, and two $\gamma$ angles. Therefore, if there exists such a vertex anywhere in a tiling, this and the tetravalent $(\delta, \delta, \alpha, \alpha)$ vertex are the only types of vertex containing $\alpha$ angles.

We start with a vertex with two $\alpha$, two $\beta$, and two $\gamma$ angles - as observed in Lemma 2.2, they must be arranged as shown in figure 32-I. The vertex $u$, which has an $\alpha$ and a $\beta$ angle, must also be of this type, and $v$ is tetravalent. We thus see that this configuration repeats, and we conclude that the vertices labeled $w$ in figure 32-II are surrounded entirely by $\gamma$ angles. Thus $\gamma=\frac{\pi}{k}$ for some $k \geq 3$, and the entire tiling, $\mathrm{N}^{k}$, is as shown in figure 32-III. 

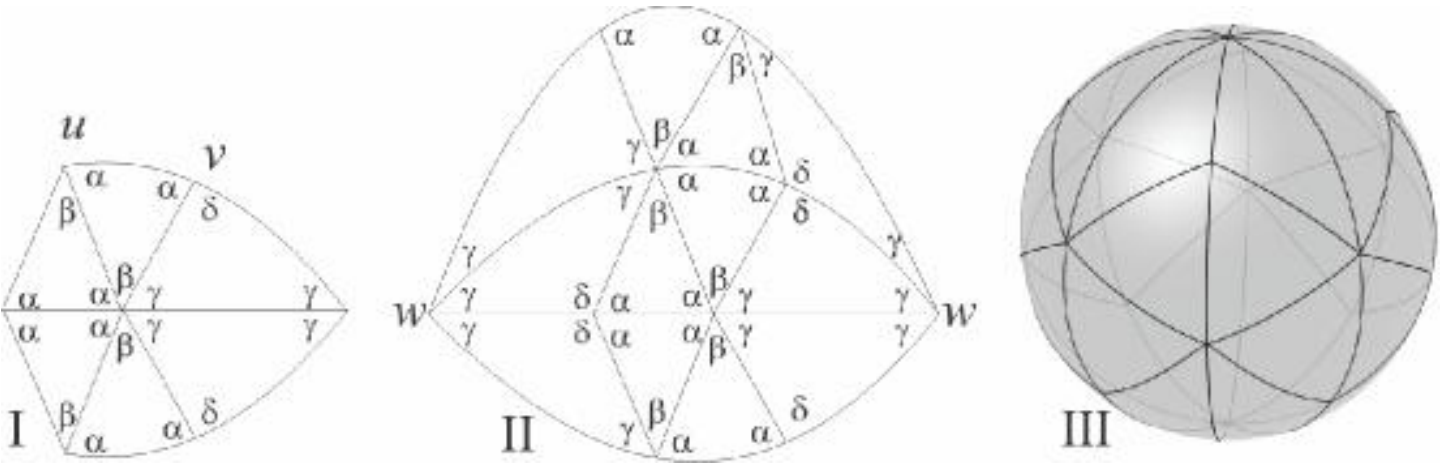

Figure 32:Case $\mathrm{C}$, and the tiling $\mathrm{N}^{4}$

We note that this tiling differs from $\mathrm{F}^{k}$ and $\mathrm{L}^{k}$. In those two tilings, the edges of the equatorial triangles lie along longitudes; in $\mathrm{N}^{k}$, it is the bases that do this.

\section{Conclusion}

This paper completes the classification, begun in [3] and continued in [4], of $f$-tilings of the sphere by two noncongruent congruence classes of isosceles triangle tiles. Those papers dealt with the cases in which the base of one triangle was congruent to the base, (resp. leg) of the other; here we consider the case in which the legs of one triangle are congruent to those of the other.

In the case where the base angles of one triangle are right, those of the other triangle must also be right. There are doubly-infinite and continuous families of such tiles, most of them giving rise to large numbers of rather similar tilings, differing only in the order of two distinct types of triangle within a "fan."

For triangles with acute base angles, on the other hand, we find either one-parameter discrete families, indexed by the number of tiles meeting in a polar rosette, or sporadic tilings that are not part of any family. One pair of tiles, with angles $\left(\frac{\pi}{3}, \frac{\pi}{3}, \frac{2 \pi}{5}\right)$ and $\left(\frac{\pi}{5}, \frac{\pi}{5}, \frac{2 \pi}{3}\right)$, tiles the sphere in millions of distinct ways, but only four of them are $f$-tilings.

\section{ACKNOWLEDGMENTS}

${ }^{1,3}$ This work was partially supported by Portuguese funds through CIDMA (Center for Research and Development in Mathematics and Applications) and FCT (Foundation for Science and Technology) within project UID/MAT/04106/2013.

${ }^{2}$ Supported partially by an NSERC Canada Discovery Grant.

\section{References}

[1] Catarina P. Avelino and Altino F. Santos, Spherical $f$-Tilings by Triangles and $r$-Sided Regular Polygons, $r \geq 5$, Electronic Journal of Combinatorics, 15(1) (2008), \# R22.

[2] A. M. d'Azevedo Breda, A Class of Tilings of $S^{2}$, Geometriae Dedicata, 44 (1992), 241-253.

[3] A. M. d'Azevedo Breda and Patrcia S. Ribeiro, Spherical $f$-Tilings by Two Non Congruent Classes of Isosceles Triangles - I, Mathematical Communications, 17 (2012), 127-149.

[4] A. M. d'Azevedo Breda, Robert Dawson and Patrcia S. Ribeiro, Spherical $f$-Tilings by Two Non Congruent Classes of Isosceles Triangles - II, accepted for publication in Mathematica Sinica, English Series.

[5] A. M. d'Azevedo Breda, Patrcia S. Ribeiro and Altino F. Santos, A Class of Spherical Dihedral $f$-Tilings, European Journal of combinatorics, 30(1) (2009), 119-132.

[6] A. M. d'Azevedo Breda, Patrcia S. Ribeiro and Altino F. Santos, Dihedral $f$-Tilings of the Sphere by Equilateral and Scalene Triangles-III, Electronic Journal of Combinatorics, 15(1)(2008), \# R147.

[7] A. M. d'Azevedo Breda and Altino F. Santos, Dihedral $f$-Tilings of the Sphere by Rhombi and Triangles, Discrete Mathematics and Theoretical Computer Science, 7(1), (2005), 123-140.

[8] A. M. d'Azevedo Breda and Altino F. Santos, Dihedral $f$-Tilings of the Sphere by Spherical Triangles and 
Equiangular Well Centered Quadrangles, Beiträge Algebra Geometrie, 45 (2004), 441-461.

[9] R. Dawson, Tilings of the Sphere with Isosceles Triangles, Discrete and Computational Geometry, 30 (2003), 467487.

[10] R. Dawson and B. Doyle, Tilings of the Sphere with Right Triangles I: The asymptotically right families, Electronic Journal of Combinatorics, 13 (2006), \# R48.

[11] Y. Ueno and Y. Agaoka, Classification of Tilings of the 2-Dimensional Sphere by Congruent Triangles, Hiroshima Math. J., 32 (2002), 463-540.

[12] Y. Ueno and Y. Agaoka, Examples of Spherical Tilings by Congruent Quadrangles, Mem. Fac. Integrated Arts and Sci., Hiroshima Univ. Ser. IV 27 (2001), 135-144. 